Open Access

\title{
Understanding openness through (in)visible platform boundaries: a topological study on MOOCs as multiplexes of spaces and times
}

\author{
Karmijn van de Oudeweetering ${ }^{*}$ (D) and Mathias Decuypere(D)
}

\author{
* Correspondence: karmijn. \\ vandeoudeweetering@kuleuven.be \\ KU Leuven, Leuven, Belgium
}

\begin{abstract}
Among new technologies in education, the Massive Open Online Course (MOOC) is one of the phenomena that received much attention. However, controversy developed around the meaning of the word 'open' as integrated in the acronym. Despite the disagreements, studies on MOOCs generally do not formulate clear conceptual or operational descriptions of what openness implies for their research. Without explicating these descriptions, it becomes impossible for HE practitioners and researchers to gain a sense of how the proclaimed open nature of MOOCs is and can be realized in practice. This study aims to respond to this debate and the prevailing ambiguity and confusion around the concept 'openness' by examining the relevance of an alternative view. That is, through the lens of social topology, this study considers how openness is enacted in multiple forms that interact. The study further examines whether and how openness is performed through the specific way in which boundaries operate. Based on an empirical case study on a MOOC platform developed and administered by several European universities, the study presents a combination of descriptions, sketches and legends that describe multiple spaces and times of openness in the MOOC platform. The study therewith provides strong arguments for the pertinence of this alternative view, and invites further research on higher education initiatives to examine the various yet specific ways openness can be established.
\end{abstract}

Keywords: MOOCs, Open education, Openness, Boundaries, Imaginaries, Social topology

\section{Introduction}

Among new technologies in education, the Massive Open Online Course (MOOC) is one of the phenomena that received much attention. Especially in media, MOOCs are framed both as a threat as well as a complement to higher education (HE), since their online and free accessibility might make them more attractive to potential students (Farrow, 2017; Weller, 2014). However, as different forms of MOOCs appeared, from community-based courses to one-to-many delivered lecture series, and from publicly funded initiatives to commercial freemium packages, more and more controversy developed around the meaning of the word 'open' as integrated in the acronym (Decuypere, 2018; Knox, 2016). In these discussions, 'openness' is repeatedly used as a spatial and temporal metaphor to discuss whether and how the proclaimed potential of

(c) The Author(s). 2019 Open Access This article is distributed under the terms of the Creative Commons Attribution 4.0 International License (http://creativecommons.org/licenses/by/4.0/), which permits unrestricted use, distribution, and reproduction in any medium, provided you give appropriate credit to the original author(s) and the source, provide a link to the Creative Commons license, and indicate if changes were made. 
MOOCs to alleviate barriers to education is enacted through their digital form. For example, one side of the debate links openness to the ambition to 'open the doors' towards educational institutions especially for those otherwise excluded from them (Weller, 2014). Digital devices are then positioned as flexible tools to counter the fixed structure of 'brick-and-mortar' and time-scheduled institutions and, by this means, to make education more accessible. From another side, openness is related to the capacity of digital devices to let knowledge 'travel' through geographically unlimited networks of individuals (Peters, 2014). Early initiators of MOOCs especially adhere to this interpretation, as they associate openness with the possibility to expand and accelerate exchange of knowledge over the Internet, while leaving room for local or personal adaptation of the subject matter (Cormier, 2008; Downes, 2007). Despite these explicit disagreements on the interpretation of openness, studies on MOOCs generally do not formulate clear conceptual or operational descriptions of what openness implies for their research (Weller, Jordan, Devries, \& Rolfe, 2018). Without explicating these descriptions, it becomes impossible for HE practitioners and researchers to gain a sense of how the proclaimed open nature of MOOCs is and can be realized in practice.

This ability to compare the announced openness of MOOCs with their actual realization is relevant, as critics have already commented that the spatiotemporal metaphors are idealized notions and omit closures that appear in practice. For example, they emphasize that online communities function around a rationale of homogeneity, which means that they are mainly 'open' to those with related interests and values yet close off to those with different views (Funes \& Mackness, 2018). Moreover, while MOOCs are openly accessible at different locations and moments in the day, they limit possibilities for contact with a teacher (Bayne, Knox, \& Ross, 2015; Knox, 2013). These nuanced interpretations of openness invite to, instead of assuming complete openness, scrutinize where and when openness is enacted, as well as where and when boundaries are crossed (Edwards, 2015; Oliver, 2015). This requires an analysis of space and time of MOOCs, which is not a straightforward effort. That is, as MOOCs relate the learners' settings with the contexts of teachers and involved institutions through a mediating interface, they build up complexes of multiple locations, distances, presences, moments and durations (Bayne, Gallagher, \& Lamb, 2014; Crea \& Sparnon, 2017; Knox, 2016). Hence, MOOCs should be understood as entanglements of multiple sorts of spaces and sorts of times. This view illuminates how the openness of MOOCs, as well as their closings, are differentially enacted through multiple interconnected configurations.

Adopting this alternative view on spaces, times and openness, this article aims to respond to the lacking conceptual and operational descriptions of openness in today's research on MOOCs (Weller et al., 2018). This alternative view diverts from the existing and highly debated conceptions of openness in several ways. First, different from other studies, this study does not aim to conceptualize, operationalize or assess openness through one or multiple separate dimensions. That is, it does not consider openness merely in terms of access nor does it divide the legal, technical or ideological aspects of openness (Ioannou, 2018; Stracke, 2018). Instead, this study intends to overcome compartmentalized notions and to comprehend how openness is enacted in multiple forms that interact. Second, the study does not assume that openness is realized through a removal of barriers (e.g., Baker, 2017). The study follows the understanding that openness arises out of practices that necessarily install boundaries in order to construct specific spaces and times. 
Accordingly, this paper aims to review the relevance of this alternative view on openness for further research and practice with MOOCs through an analysis of spaces and times within a particular MOOC platform. The first part of this paper discusses central premises of the theoretical and methodological approach that grounds this view, social topology, an approach that is gaining increasing interest in educational research. Then, the paper draws on a topological conceptualization of openness, i.e. 'the open' as 'the visible, to sketch how openness can be understood in relation to what is made visible and invisible. Based on these ideas, it is argued that spaces and times, and thus openness, can best be understood by focusing on boundaries that distinguish between what is seen and what is not seen. Then, it is explicated how these arguments inform the methodology of this study on a selected MOOC platform. Specifically, the data collection and analysis focus on the distinction and relation between the visible appearance of the user interface and the 'invisible' infrastructures beyond this interface. Structured by this dual focus on 'on-interface' practices and 'beyond-interface' practices, the reported findings specifically describe how multiplexes (i.e. multiple interconnected forms) of spaces and times are produced through boundaries that make certain practices either visible or invisible, with a particular interest in how this establishes openness.

\section{Conceptual framework}

\section{Social topology}

In everyday life, space and time are usually indicated through external measures, like locations are pinpointed on a map or seconds counted on a clock. However, not all phenomena can just as easily be 'located' through these measures. This is equally the case for websites, as they cannot be fixed to a particular place, nor can they be related to a stable beginning or end time. Social topology as a theoretical and methodological approach aims to understand these more complex spaces and times and, rather than merely typifying them through external measures, it considers their internal characteristics as well (Martin \& Secor, 2014; Mol \& Law, 1994). That is, social topology recognizes how spaces and times are composed of relations. These relations make up practices through their specific configurations. A topology, then, is a set of practices that produces spaces-times (e.g., Mezzadra \& Neilson, 2012). Moreover, social topology considers how spaces-times depend on each other. This relates to the often-cited example of 'time-space compression', which points at the way that technologies accelerated communication and changed the presence of distant entities, producing a nearness that again intensified time (Harvey, 1989). As shown in this example, social topology highlights how complex spaces-times are neither pre-existing 'containers' that can always be measured nor merely mental constructs, but are produced through concrete practices (Tucker \& Goodings, 2014).

Social topology is inspired by, and builds on, the premises of topology as a sub-field in mathematics. This branch of mathematical studies introduced the idea that spaces can be examined through relations that take form as figures, like circles, cubes or manifolds. A mathematical topological space is specifically characterized by relationships that are continuous under transformation. For example, a circle can transform into a square without disrupting the intrinsic relations or lines that build up the figure, whereas introducing a separating line into the circle does mean a break with its 
continuity. This means that topological spaces are established within figures that twist, fold or extend without breaking or melting (Sha, 2012). Social topology largely adopts these premises of mathematical topology, and approaches settings or objects as figures that make the continuous relations that constitute topologies apparent (Lash, 2012; Martin \& Secor, 2014). This focus on continuity also helps to identify what is and remains specific to the relational configurations, and therewith to see what is valued or what are the norms within these configurations (Decuypere \& Simons, 2016). In other words, social topology recognizes how spaces-times operate as imaginaries and thus, that they are not 'imagined realities' but they are normative views established through shared concrete practices (Jasanoff, 2015; Ross \& Sheail, 2017). Different from other perspectives in educational research, social topology can therefore serve to interpret from empirical accounts how practices are related to ideas about what education is or should be. In this way, the approach can equally help to trace what and who produces these norms, and how these norms have become stabilized (Lash, 2012; Marres, 2012).

Given the complexity of social relations, social topology considers how multiple topologies produce different spaces-times that continuously overlap and intersect with each other (Mol \& Law, 1994). Even within one setting like a classroom, different spatiotemporal forms co-exist and are interrelated as practices between teachers, students and materials are made up of different relations (Sørensen, 2009). This means that, besides the fact that social topology can help to develop a characterization of openness without ignoring the normative nature of a particular definition, it equally can serve to how openness can be envisioned otherwise as they can be established in multiple forms (Martin \& Kamberelis, 2013; Thompson \& Cook, 2015).

As social topology grasps multiplicity, complexity and specificity of spaces and times, it is particularly suitable for studying the realization of openness in online spaces of MOOCs. For example, a social topological approach acknowledges how websites continuously reconstruct spaces and times through their interactive nature and how they, by this means, counter the imagery of a fixed and pre-determined space as established in immutable media like print (November, Camacho-Hübner, \& Latour, 2010). It further addresses how online learning becomes a complex amalgam of practices that involve studying, as well as work, family and travel, and how this produces specific spaces and times (Sheail, 2018). However, topological studies do not and should not merely address visible practices, as topologies in websites also involve algorithms, codes and material infrastructures that remain 'hidden' on website interfaces (Kitchin \& Dodge, 2011; Knox, 2016; Parisi, 2012). In order to understand spatiotemporal configurations of websites, including openness, it is necessary to examine the visible as well as the invisible features embedded in them (Edwards, 2015).

\section{The 'Open', the visible and its boundaries}

The importance of visible and invisible practices for a topological understanding of openness is addressed by Deleuze (1986), as he positions 'the Open' as 'the visible'. The open here refers to the visible explication of practices that produce topological imaginaries. For example, when or if one thinks of school, these ideas are mainly built on the visual encounter with a school, its architecture, its learning and teaching materials and its time schedules. While texts and words can describe these imaginaries, Deleuze highlights how a textual description only acts on the visible. Hence, visible entities have a 
particular quality to establish conditions as well as to set limits to what we have words for, while language builds relations between and with visible entities. This intricate relation between language and vision is why the Visible or the Open does not give us something to see without also providing something to speak' (Deleuze, 1986, p. 111). Following this argument, the visible or the 'open' determines what we can see and speak about. More importantly, particular relations with words delimit and normalize how we can think about what we see and, in this way, produce imaginaries. For a school as exemplified above, as well as for a digital form of education such as MOOCs, this means that visible arrangements like the architecture or learning materials establish norms on how to think about the practice of schooling. What is closed off from visibility, is positioned beyond the boundary of the imaginary and thus, 'outside' of our norms.

This conceptualization describes how openness as performed by 'the visible' refers to more than what is 'open to sight', for example on the interface of MOOC platforms. Specifically, this conceptualization relates what is made open to sight to what is included in and what are the boundaries of our imaginaries, and thus, how normative views become made and delineated. Boundaries can therefore be understood as productive of shared spaces and times as they select and order what and who is included in our collective imaginaries (see also Burridge, Gill, Kocher, \& Martin, 2017; Mezzadra \& Neilson, 2012; Thompson \& Cook, 2015). Boundaries can be considered to produce shared spaces and times in another way as well. That is, their position between localities makes that they can, and often do, mediate practices with 'adjacent' localities (Star, 2010; Star \& Griesemer, 1989). A website, for example, constitutes a boundary of and between what a user can see (e.g., front-end interface, immediate 'user environment') and what a producer can see (e.g., back-end work). Yet the website also constructs the space they share, and through which their presence becomes visible 'on the boundary' (e.g., users see the website as created by producers; producers see data of users' navigations). It can then be considered that this shared space enacts an 'openness' as it mediates between localities otherwise closed off from each other. These considerations emphasize that openness is performed by particular workings of boundaries, rather than an absence of boundaries. In order to understand openness, it should therefore be understood what boundaries open up for, what they close off and how they relate different localities.

\section{The examined figure and the topological methodology}

The figure of this study, the MOOC platform that is analyzed, is named 'European Multiple MOOC Aggregator' (EMMA). EMMA is administered by several European universities and financially supported as a pilot project by the Competitiveness and Innovation Framework Programme (CIP) of the European Commission (EC). In their publications, initiators of EMMA formulate four explicit accounts of openness to which the platform relates. That is, openness in EMMA is related to (1) access to the course content (2) learning in networks (3) learning across European national boundaries and (4) data, research and experimentation (Kerr \& Eradze, 2016). The project initiators further explicate several platform features that should aid the realization of these accounts. First, they emphasize that the platform provides free-of-cost and creative commons licensed course material and stimulates learning in networks of peers (ibid). Furthermore, MOOCs are provided by various European teachers and universities, using a system that automatically translates course content into 
different European languages. Moreover, a personal learning environment (PLE) is developed to help learners integrate materials from these diverse MOOCs. Finally, multiple research methods are employed to monitor MOOC pedagogies and their impact (Kerr \& Merciai, 2015).

The platform is studied through a methodology that is developed to closely align with the topological conceptualization of openness and its relation to visibility and boundaries. Understanding boundaries as shared spaces that mediate between localities invisible to one another (Star, 2010), the interface of EMMA is considered a central boundary. That is, producers as well as users situated in different localities conjointly produce spaces and times as visible on the interface. Interested in its workings, the study aims to move and situate itself on and beyond this boundary (cf., Mezzadra \& Neilson, 2012) . Along these lines, a distinction between on-interface practices (i.e., visible for website visitors) and beyond-interface practices (i.e., visible in professional work on EMMA) guided the methodology of the study.

To examine on-interface practices of EMMA, data were collected through an active navigation of the platform. This means that the first author of this article, for about 4 weeks, visited the EMMA website and carefully examined the homepage, informational pages, the registration procedure and 6 MOOCs of different universities and from different countries. This was documented through video screen recordings and written reflections. Because funding and production work on EMMA was paused at the time of the navigation, all MOOCs on EMMA were presented in a 'self-paced' mode, i.e., archived and without continuing interactivity between learners and teachers. Hence, interactions between learners were only examined as documented, rather than 'live'.

After the navigations, the same researcher conducted interviews with EMMA team members, all involved in the development and maintenance of the platform. These interviews served to examine 'beyond interface' practices and covered their daily work, sociotechnical infrastructures of EMMA and, depending on the role of the interviewee in EMMAs development, the educational philosophy and the collaboration with the EC. The interviews were recorded, transcribed, and complemented with notes. Valuing a dialogic stance towards ethics, agreement and understanding of the participants' concerns was continuously sought. This means that, besides adopting a consent form to safeguard their informed and voluntary participation, we ascertained participants' confidentiality and anonymity and prioritized multiple member checks.

Data resulting from the navigation and interviews were brought together and reconstructed to present multiple topologies in the figure of EMMA. The data were analyzed inductively and the four accounts on openness proclaimed by EMMAs initiators were considered relevant starting points to identify and conceptualize topologies as network, access, European and data topologies. The subsequent sections of the paper introduce descriptions on how these topologies are produced, for each topology starting with the operations of on-interface practices followed by explicating beyond-interface practices. To illustrate practices on the interface, screenshots of the website are presented. ${ }^{1}$ In addition to these images, sketches on the forms of the topologies, i.e., the way they configure relations, are presented. In these sketches, a circle presents boundaries of spacestimes 'around' EMMA. The circle, as the only form that holds continuous distance between a center and the perimeter, helps to visualize how different actors can be connected to each other and through a center without presenting different intervals or 
distances between them. Through lines and other graphical shapes, as well as through accompanying legends, the specificity of the relational distributions within the topologies are further sketched and explained. Although they do not cover all practices, the combination of descriptions, sketches and legends approach a comprehensive explanation of specific spaces and times operating in the figure of EMMA.

\section{Network topologies}

By referring to networked learning (Kerr \& Eradze, 2016), initiators of EMMA relate to the spatiotemporal conception that online networks in MOOCs connect geographically dispersed individuals (e.g., Downes, 2007). In the figure of EMMA, network topologies indeed appear to connect individuals, but they do not connect anything, anyone, anytime and anywhere. In what follows, it is described how these topologies are produced by on-interface practices that attach learners and teachers as fragments and through spatiotemporal boundaries. Consequently, beyond-interface practices that establish hybrid knowledge and common interests, as well as workflows are illustrated as network topologies. Both kinds of practices show to operate around rationales of stabilizing constructions and co-presence. Therewith, they selectively tie together individuals and entities to establish specific configurations or forms of relations.

\section{On-interface: attachments}

Attaching fragments On the EMMA interface, networked relations particularly come forward in webpages that enable interactions between learners and teachers. These interaction pages are named 'Conversations' (i.e., text boxes with comments and replies on lesson pages; see Fig. 1), 'EMMA posts' (i.e., pages where all learners and teachers

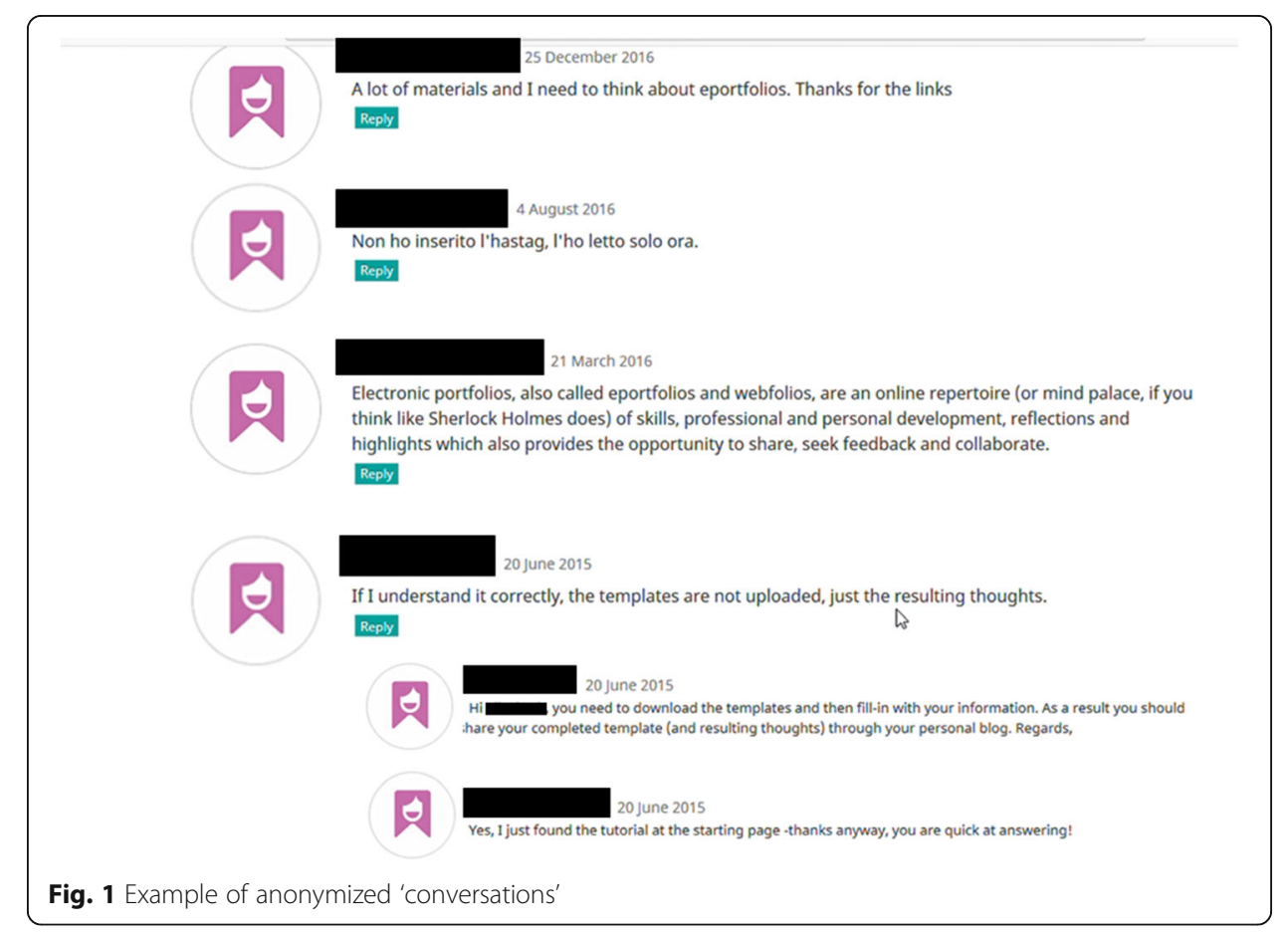


can post and reply) and 'virtual classrooms' (i.e., similar to EMMA posts, yet only accessible for learners and teachers in the same MOOC). Furthermore, learners can interact with each other on dedicated Twitter and Facebook pages presented through hyperlinks in the majority of MOOCs. All these pages follow a particular structure: contributions of learners and teachers are delineated in comment- and reply boxes, are labelled by a name, a photo and a date. The comments are ordered chronologically, which creates a sense of linear time, yet it also emphasizes the asynchronous nature of the interactions (Bødker \& Brügger, 2018). Moreover, the lines around the text boxes and the individual 'signatures' withhold contributions to merge into a different form like, for example, a Wiki. Thus, although the interaction pages enact shared spaces in which learners can act and communicate, the specific structure also illuminates that they act separately without being able to make adjustments to this separation (cf., Star, 2010). This space-time establishes an imaginary of learning as a practice of attaching pieces of information on a common surface, instead of attaching (i.e., relating) learners to each other. This network topology therewith presents itself as a spatiotemporal formation, a stabilizing construction so to say, in which the boundaries bring learners and teachers together in a coherent frame while remaining fragmented (Fig. 2).

Attaching through spatiotemporal boundaries Overlapping with this construction is a network topology that is enacted through release and closing dates of MOOCs and their lessons. That is, traces on the EMMA interface show a high peak of interactions within particular periods and on particular pages, namely, those of page-courses that are announced to be 'active' and closing soon (see Fig. 3). Initially, these announced dates help to locate on what pages their teacher and peer learners are present and in what week, in order to know where and when to ask them questions or finish collaborative assignments. Even though MOOCs are still 'open' in a self-paced mode after the deadline, the

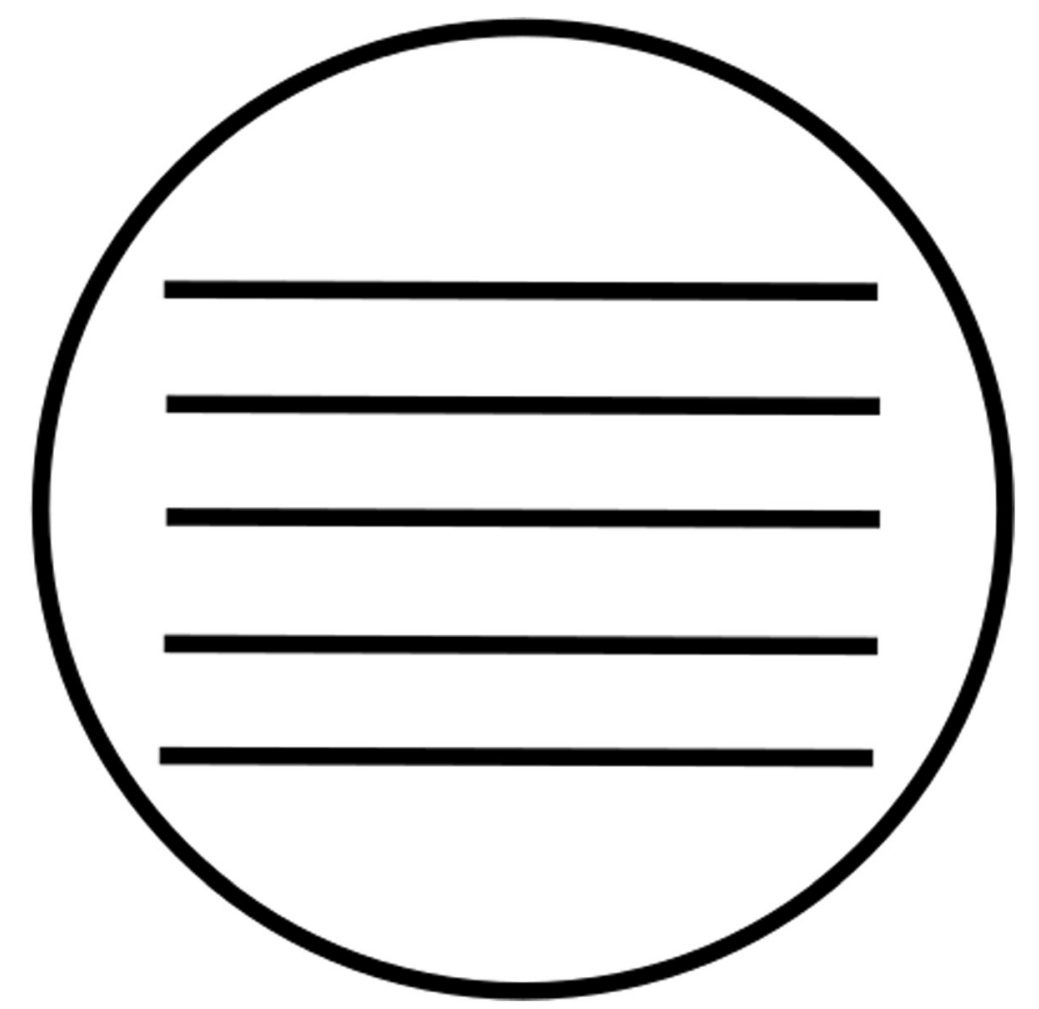

Fig. 2 Legend: The horizontal lines present the fragmented contributions of learners inside EMMA 


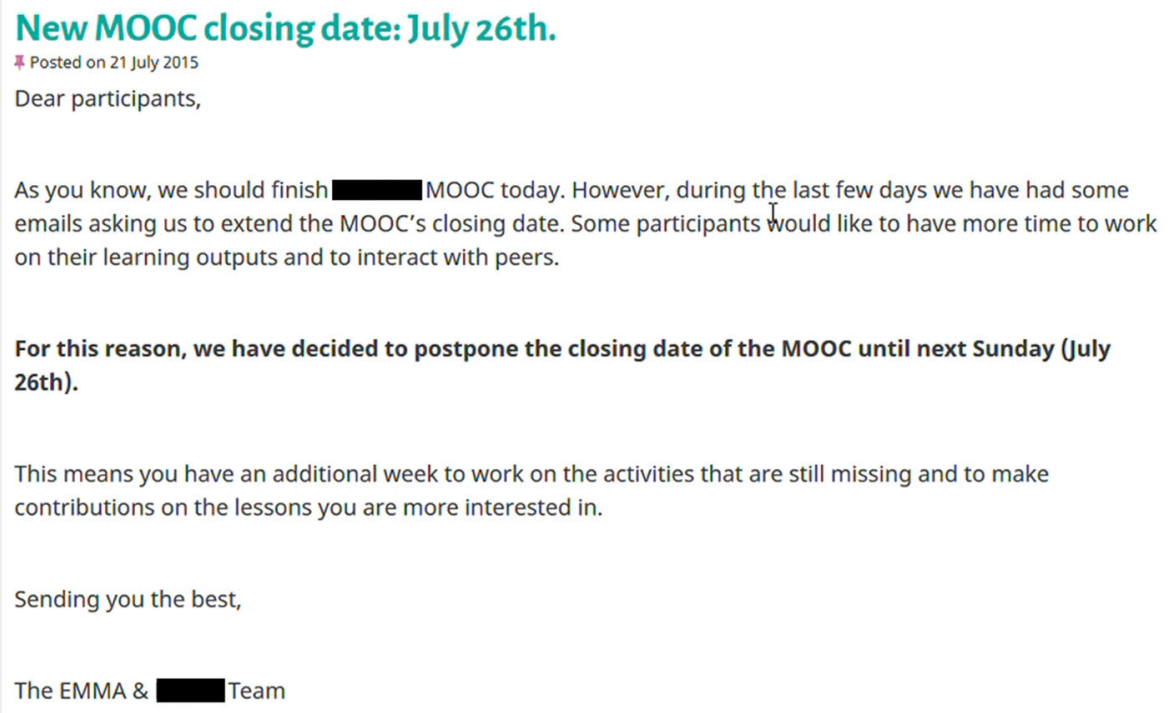

Fig. 3 Announcement of a closing date of a MOOC

announced dates operate as spatiotemporal boundaries that establish a 'current time' or a 'synchronous' time that is typical for a classroom (Bødker \& Brügger, 2018; Leaton Gray, 2017). This network topology is, thus, spatiotemporally forming a co-presence and bounds a common path with shared spaces-times in which learners can attach to each other rather than merely attaching to the same surface (Fig. 4).

\section{Beyond-interface: on the same page(s)}

Hybrid knowledge and common interests Moving beyond the interface, networks are made up by relations between EMMA associates ${ }^{2}$ that generally remain invisible on the screens of visitors' devices. These associates mainly comprise institutions that provided MOOCs (i.e., providers), partners that cooperated in developing platform features (i.e., partners), the EC as the funding institution and the EMMA team. Because the development of the website remained contingent upon the relations among associates, the EMMA team had a daily task in maintaining these relations through recurrent contact. As some of the associates worked on the communication side, some on the educational design while others on the data processing and analysis, the EMMA team had to negotiate between different forms of knowledge. In order to do this, they worked with 'hybrid' knowledge that helped them integrate the 'local languages' (i.e., jargon of the different associates) into the general objectives of EMMA (Callon, Lascoumes, \& Barthe, 2009; Star \& Ruhleder, 1996). For example, team members with didactic responsibilities translated their background knowledge into ideas for digital educational tools, whereas IT staff members merely used their knowledge on coding languages to develop these tools. Subsequently, teachers were able design their MOOC according to their knowledge 


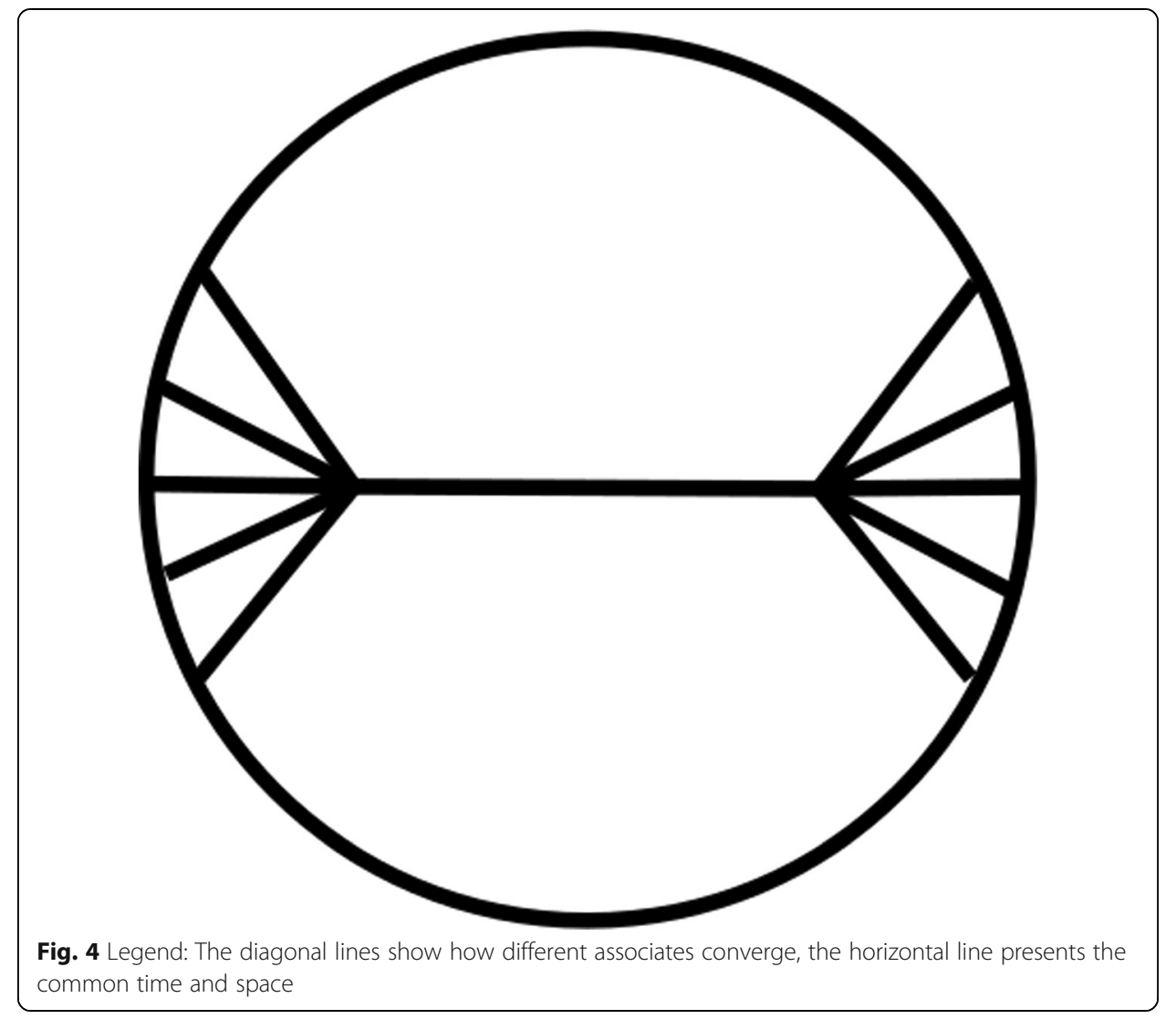

on the subject matter and based on their educational objectives with a 'ready-made' platform, not knowledgeable of its underlying code. This means that team members were continuously working on boundaries rather than working to overcome boundaries. However, these boundaries enact shared spaces in which they could work together without common knowledge yet with a common interest (Star, 2010; Star \& Griesemer, 1989). This made that the formation of relations appeared as a stabilizing construction, yet in a different form than on the interface. That is, all associates were connected through a common interest, and where able to adapt the construction from their own disciplinary viewpoint (Fig. 5).

Workflows Networked relations beyond the interface are also performed through particular tools. One tool was the 'Gantt chart', a timeline in which 'work packages' (i.e., clusters of tasks) and 'deliverables' (i.e., planned outcomes of the work packages) are ordered. Another set of tools comprised different kinds of software, such as those for project management, file sharing, telecommunication and slide presentation. As one of the team members noted, both configurations made sure associates were 'on the same page'. In other words, similar to the establishment of release- and closing dates on the interface, these beyond-interface practices helped to locate 'who' was present 'when' and 'where'. In this instance, the boundaries helped to locate associates' presences 'in' work packages, in tasks, and within working files. However, the two sorts of tools operate in different ways. The Gantt chart 


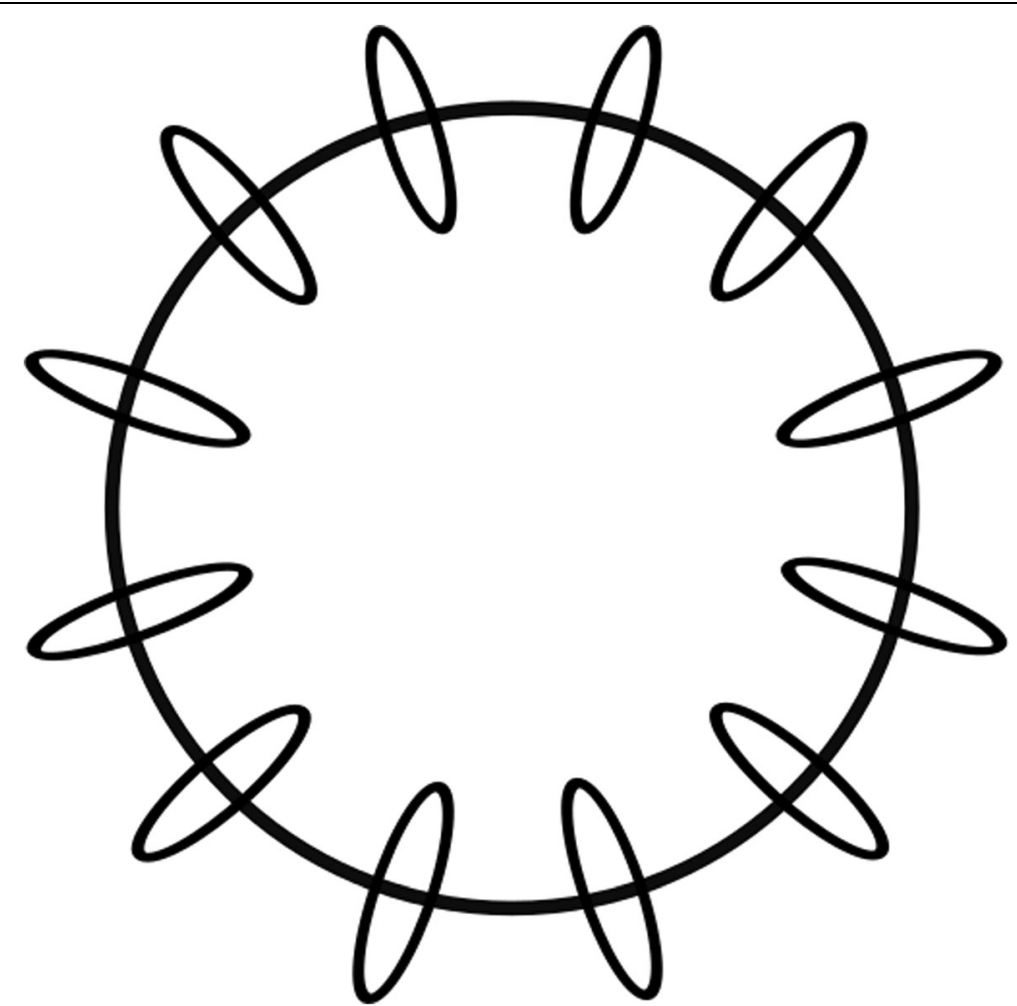

Fig. 5 Legend: The rings present EMMAs associates, as they are collectively shaping, working on and connected through the boundary as a shared space

as an orderly timeline enacts an imaginary of EMMAs development as a linear process, which clarifies the collaborative workflow and can be shown to a wider public, including website visitors and the EC (Alhadeff-Jones, 2017; Latour, 1986). Unplanned and less linear work, however, was only appearing in the software that was hidden from the 'outside world' (Star, 1999). Hence, these overlapping topologies are performed by boundaries that separate spaces and times for those producing the platform and those who are seeing the platform (Fig. 6).

\section{Access topologies}

Reflecting broader discussions on MOOCs, initiators of EMMA relate openness in terms of access to the ubiquitous, free-of-cost and creative commons licensed nature of its learning content (Kerr \& Eradze, 2016). However, as access actually implies a means of entry, it should not only refer to what we have access to but equally to what we have access through. As described here first, access topologies in EMMA comprise on-interface practices that perform entrance, and that subsequently enact a path similar to a traditional school, yet equally a digital school. Second, it is explicated how beyond-interface practices perform access topologies, establishing the platform as a mutable space and as one that centralizes learners. Together, these topologies select and order relations through boundaries. As the access topologies appear to act upon constructions and co-presence, it is shown how they intersect with network topologies. 


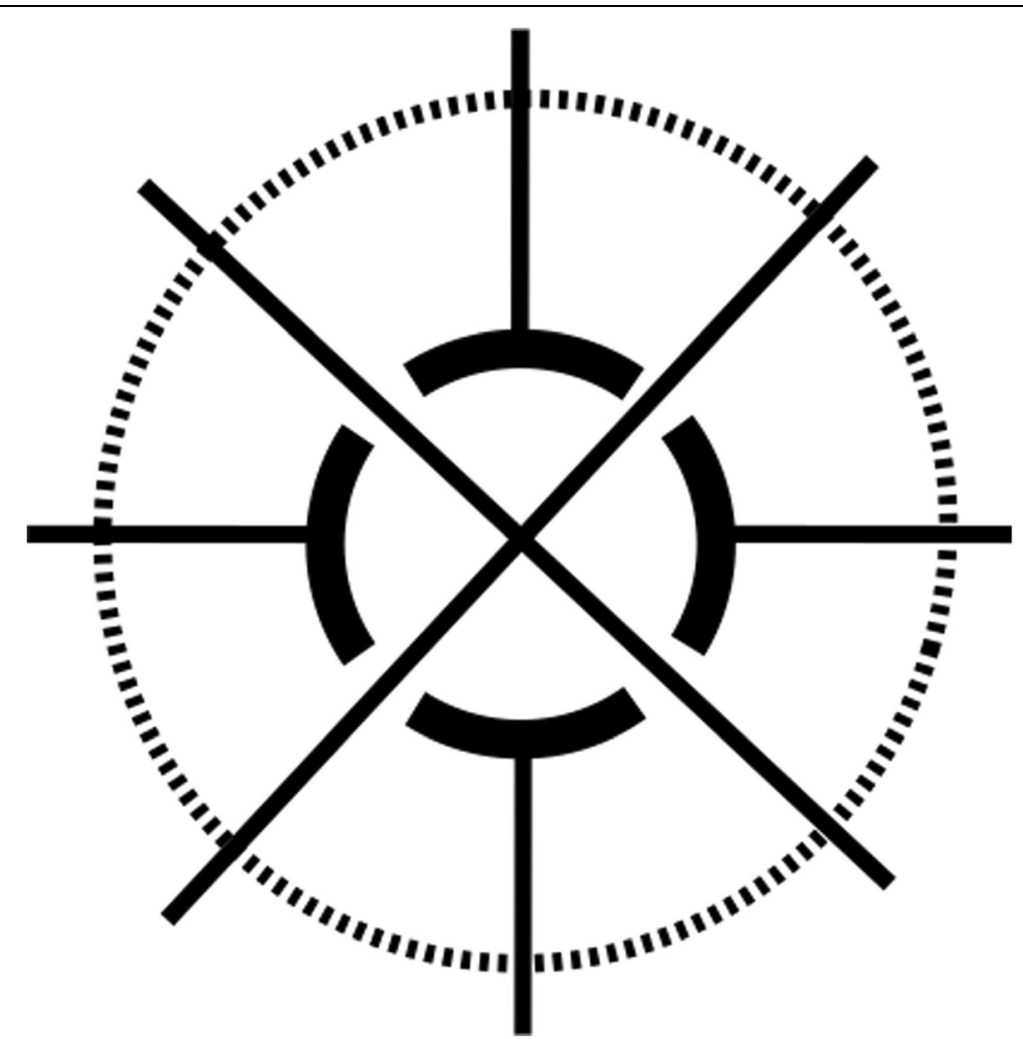

Fig. 6 Legend: The porous circle indicates EMMAs shared space established through the Gantt chart, open to those visiting the platform (horizontal and vertical lines), whereas the inner circle with four 'gaps' presents the selective shared space of the collaborative software, merely including the associates (diagonal lines)

\section{On-interface: passing through the school infrastructure}

Entrance On the interface of EMMA, access topologies stretch over multiple webpages that are all connected to the homepage. Hence, the URL to the homepage can be understood to operate as an 'outer boundary' or, more precisely, as a first access point or an entrance (cf., Star, 1999). The homepage shows a photo of three young individuals holding digital devices within a sketch of an urban environment, combined with the motto 'MOOC different!' (see Fig. 7). Through the text, MOOCs on EMMA are positioned as 'different' from 'others', a position that enables to form a distinct identity (Srinivasan, 2017). The adjoining photo then relates to an imagined learning audience with particular characteristics (e.g., age, background, taste). This combination of text and image thus establishes a selective imaginary, where EMMA provides entrance to a targeted group of learners.

A second selective imaginary appears through another entrance, namely, registration. Registration, as a requirement to become a learner, orders personal information including names, email addresses, birth dates, spoken languages, geographic locations, and optionally gender and professions. Moreover, it requires consent to policies and conditions, including consent to data collection and conformation to certain behaviors. Here, registration sets preconditions for being 'in' EMMA and therewith selects and orders the entrance further (cf., Burridge et al., 2017). Specifically, the platform itself only opens up to the learner if and 


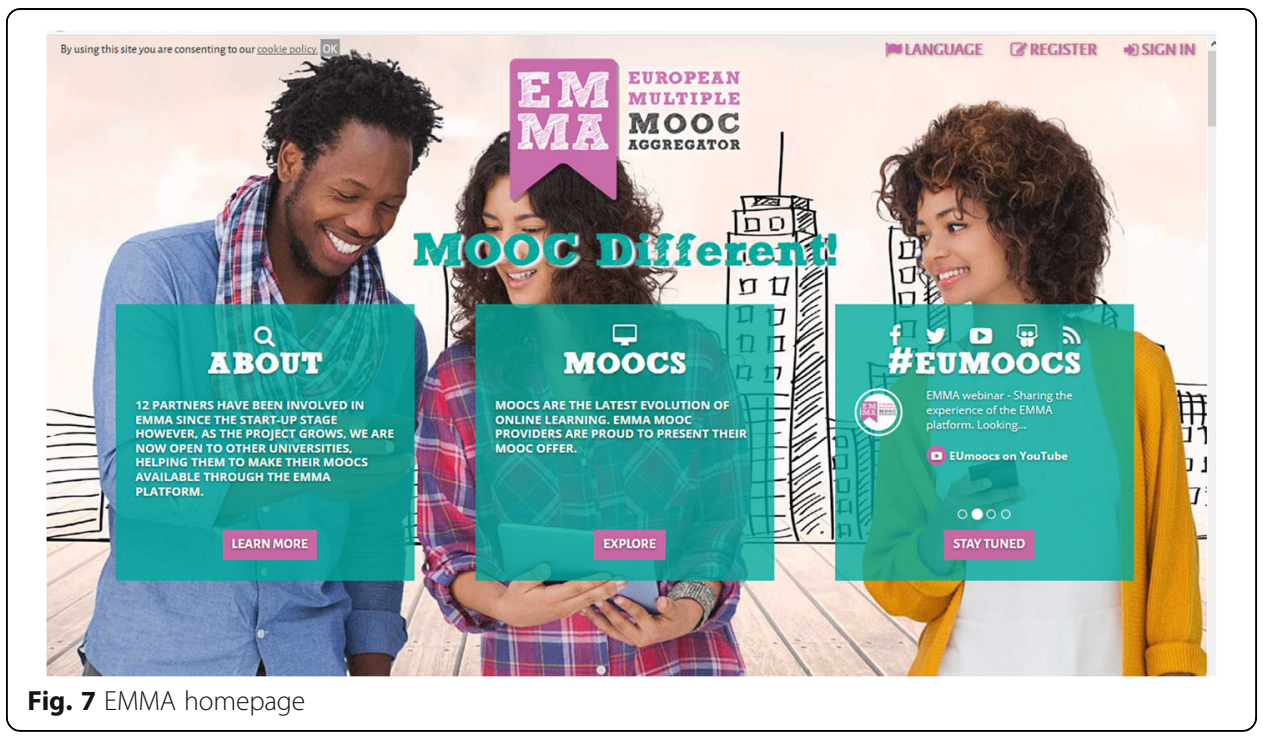

after the learner is willing to be 'open' as visible towards and within the platform (Decuypere, 2018). Together, these imaginaries produce access topologies that select who can access through EMMA, particularly through the performance of boundaries.

Traditional school A new topology emerges as a series of 'access points' after registration. That is, a series of hypertextual images is presented that shows titles of MOOCs, names of providing universities and release dates, and while hovering over also presents teachers' names and a small introductory text. By granting partial insight into MOOCs, these hypertextual images can be seen as 'windows' to different classrooms. Through a click, and subsequently through the 'enroll' button, learners can access the MOOC lessons and units that can then be followed through 'next' and 'previous' buttons. By this means, hyperlinks enact boundaries similar to walls and time schedules in a traditional school, as they guide a sequential path through an entrance, through registration, through hallways, through classrooms and through a series of lectures. The spatiotemporal formation of the relations is therefore one with multiple ordered paths, all coming back to the same initial entrance (Fig. 8).

Digital school Contrasting this school imaginary, an overlapping topology produces access through the website in a less sequential order. That is, within each lesson, the EMMA learner is constantly directed to and through different pages designated for particular activities. For example, learning materials and assignments often appear on external websites or software programs and learners need to go to a separate personal page called 'coursebook' in the personal learning environment (PLE) to see their notes. While hyperlinks establish connections between these pages, they also establish a separation of different spaces (Dahlberg \& Bagga-Gupta, 2017). This separation is enacted through the absence of simultaneous visibility, that is, an overview of the different activities. The combination of connection and separation, here, establishes a fragmented space-time configuration typical to a digital school (Hassan, 2017; Leaton Gray, 2017). The spatiotemporal formation of learning within this topology is characterized by breaks, turns and 'blind alleys' which forms relations like a maze (Fig. 9). 


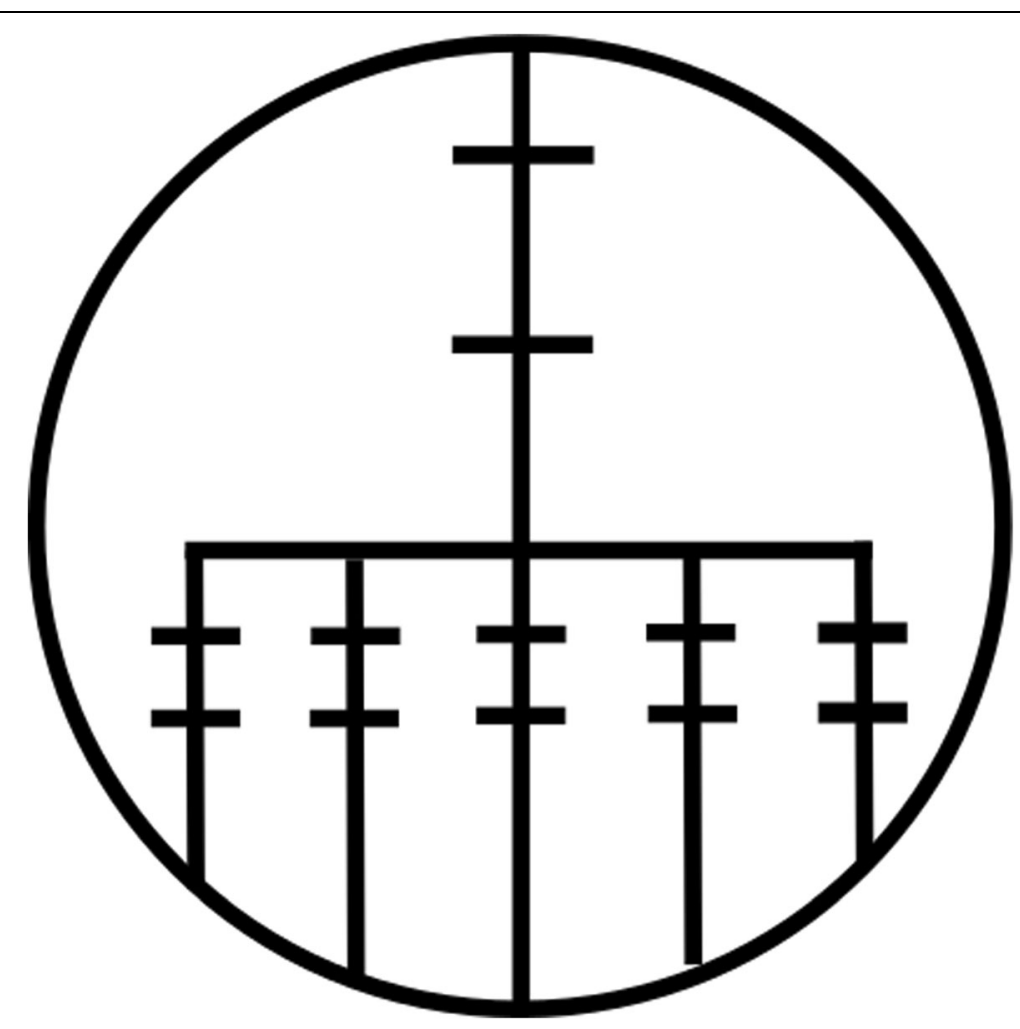

Fig. 8 Legend: The horizontal lines indicate access points, the vertical lines illustrate the linear access through EMMA

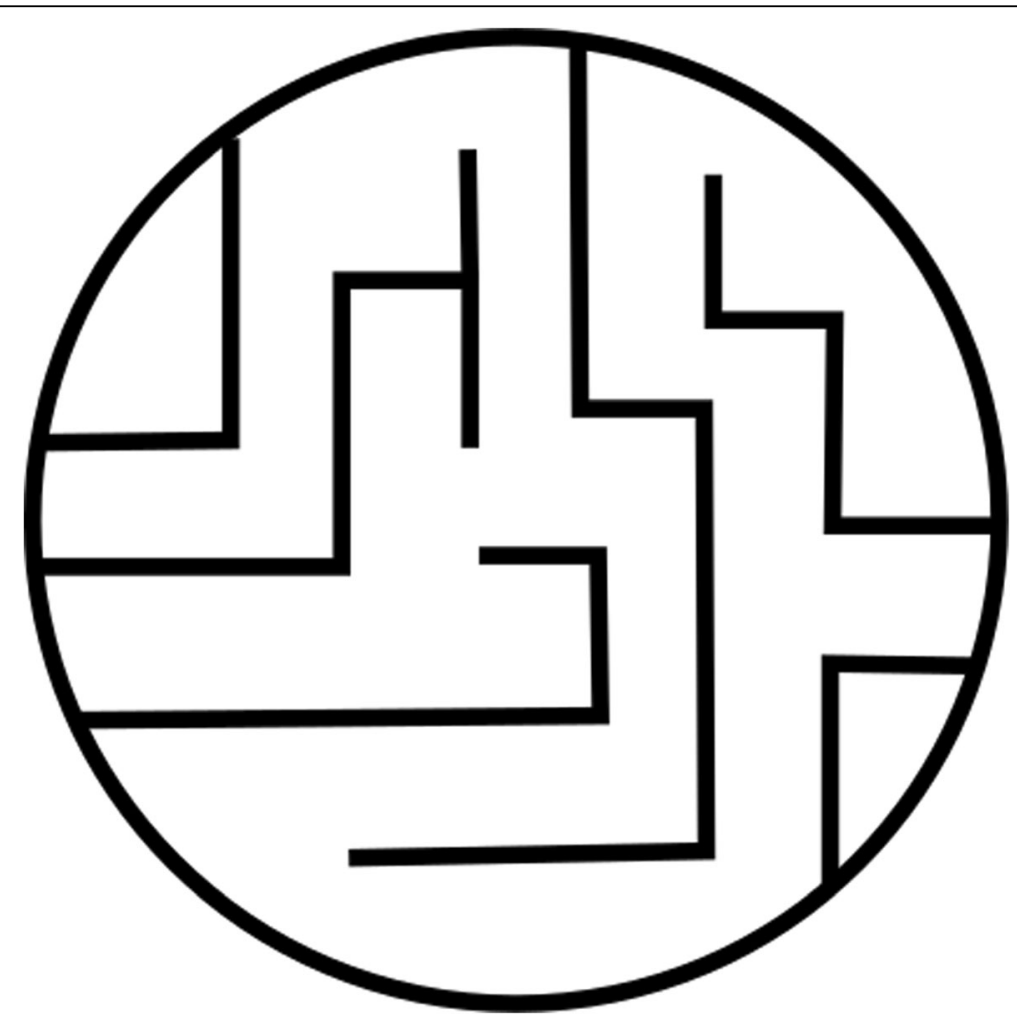

Fig. 9 Legend: The unpredictable and discontinuous connections are illustrated through the maze-like lines 
Beyond-interface: hosts and backend access

Mutable space Beyond EMMAs interface, access topologies are enacted by a wide infrastructure that generally remains hidden for website visitors. However part of this hidden infrastructure, the webserver made its central role visible in moments its operations stopped or altered (Star, 1999). First, the webserver showed itself when a MOOC with 'live' lectures suddenly became popular and it needed to be upgraded in order to generate sufficient space for these large synchronous online gatherings. Later, the webserver's size was reduced again due to budget cuts, which manifested in longer page load time and it could provide access to fewer visitors. Besides staging the webserver's operation as a host, i.e., establishing access through EMMA for website visitors, these moments show how the webserver enacts the imaginary of a mutable space as it forms EMMA in different sizes and speeds (Harvey, 2012; Ruppert, 2012). The webserver's operations therefore do not change the relations that make up spaces and times of EMMA, yet it makes the figure of EMMA appear in a continuously stretching and shrinking form (Fig. 10).

Centralizing learners Hidden for website visitors, yet specifically visible to teachers and other EMMA associates, is the access enacted through 'back-end' interfaces. EMMAs back-end allows teachers and team members to post their contributions with a personal photo and thus, to establish a presence or a co-presence on the front-end together with learners (see Network topologies). Yet the back-end also embeds a

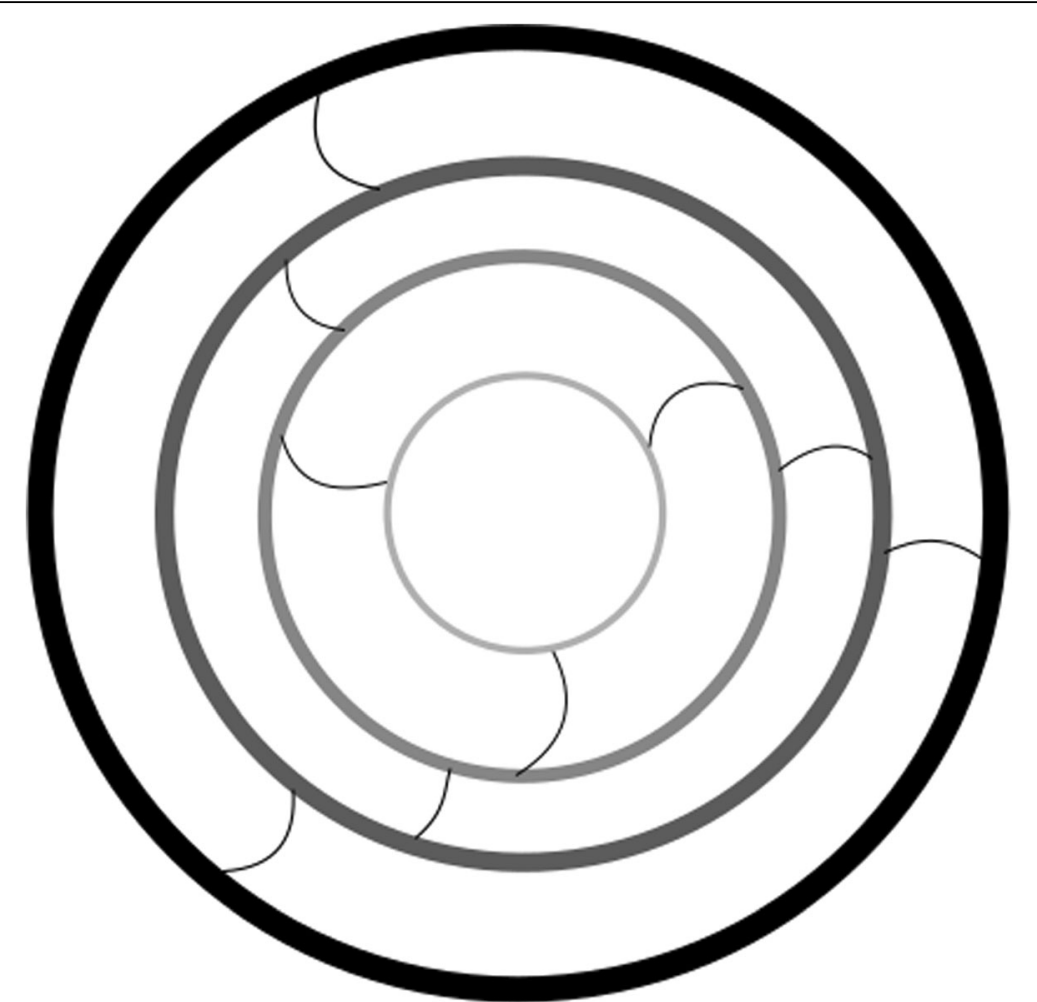

Fig. 10 Legend: The arrows indicate the increasing and decreasing movements of the webserver; smaller circles indicate the different sizes and speeds of EMMA. 
notification system that visualizes questions and comments of learners within a single pane instead of scattered over multiple pages as on the front end. This produces a new topology that not only intersects with networked co-presence, yet equally centralizes learners in order to enact access to learners. More specifically, instead of connecting learners and teachers among each other, the visualizations on the back-end establish a one-to-many relationship where teachers and team members could get access to learners through a centralized pane (Fig. 11).

\section{European topologies}

While EMMA explicitly aims to transgress European national borders through its online delivery and multilingual content, this position equally substantiates the existence of boundaries that mark European territories, i.e., geographic regions claimed by legislative power of nation states or the EU (Burridge et al., 2017). Hence, EMMAs narrative presents a complex interweaving of digital, linguistic and territorial spaces. First, oninterface practices of EMMA that produce European topologies are presented, as they draw borders around and within Europe through various symbols, they enact home countries and a space-in-between through linguistic practices and finally show how learners slip through particular borders. Subsequently, beyond-interface practices that enact a conflated Europeanness and linguistic bridges are laid out. Together, these European topologies interweave with digital, territorial and linguistic practices and as a result, produce boundaries that include and exclude territories and operate as shared spaces between different localities.

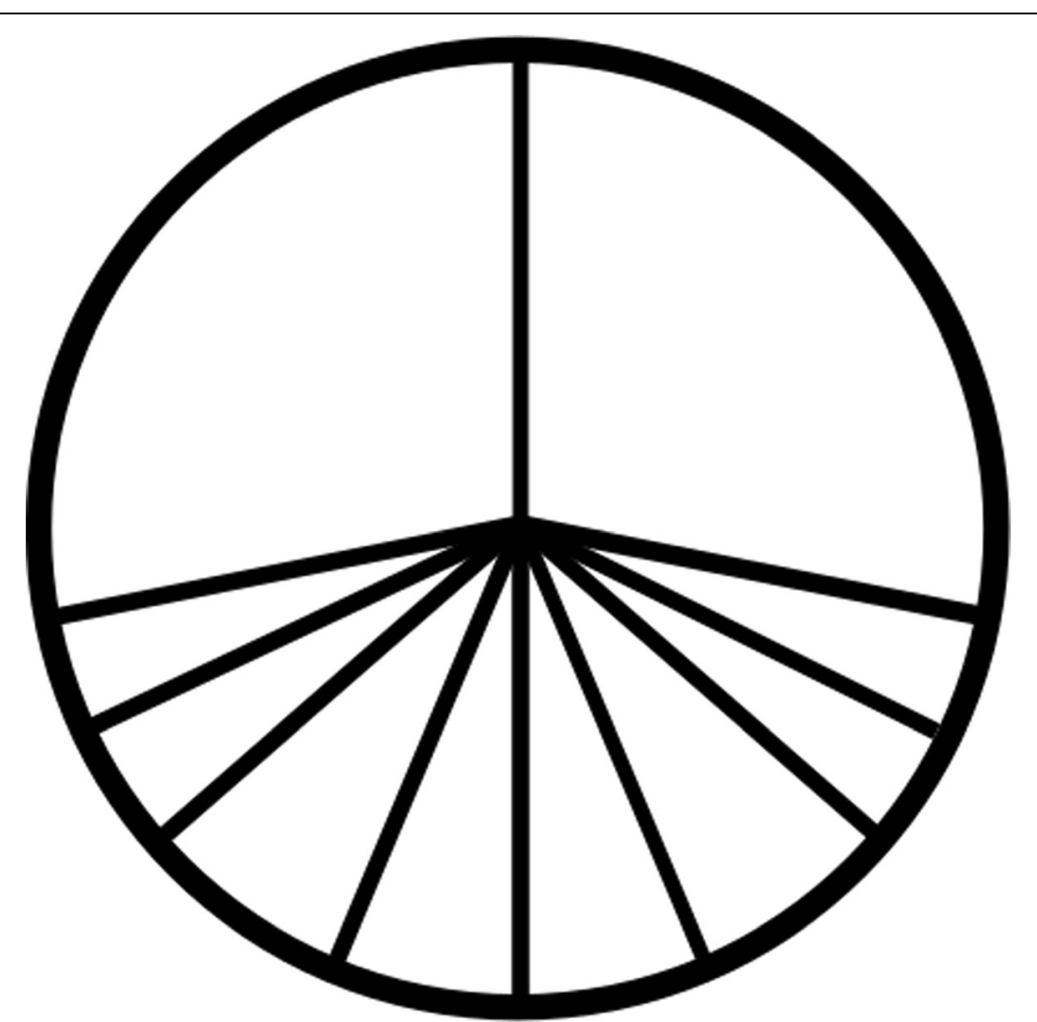

Fig. 11 Legend: The vertical line illustrates the access to learners from back-end, the diagonal lines illustrate the centralization of learners in this backend 


\section{On-interface: territorial borders of the screen}

Borders around and within Europe On the EMMA interface, several practices apparently perform spaces-times of Europe. For example, through an image of the European flag on the bottom of the home page and next to the EMMA logo in video lectures, the EU is presented as a 'grounding' and closely affiliated institution of the platform. Moreover, outbound links to proclaimed 'Related initiatives' all lead to websites or platforms released under EU support, designating a European field of online education. In this way, EU member countries are not only bound together, but they are also separated from 'outside' countries (cf., Büttner, Leopold, Mau, \& Posvic, 2015). Specifically, this performs an imaginary of Europe with boundaries around EU territory. Other practices, however, emphasize boundaries within the EU area. For example, the pictogram of a neutral flag combined with a text button 'Language' signifies a drop down menu for the selection of nine available languages on the platform (Fig. 12). The combination of this image and text reinforces the association between language and countries or regions (viz., Catalan is included as a regional language), while the selective range of languages additionally shows how the platform includes as well as excludes specific regions in the European imaginary (Opitz \& Tellmann, 2015). This establishes an ambiguous spatiotemporal formation that marks a difference between what it means to be 'in' Europe, 'in' a European field of online education and 'on' EMMA, making it possible to be included and at the same time excluded through multiple boundary systems (Fig. 13).

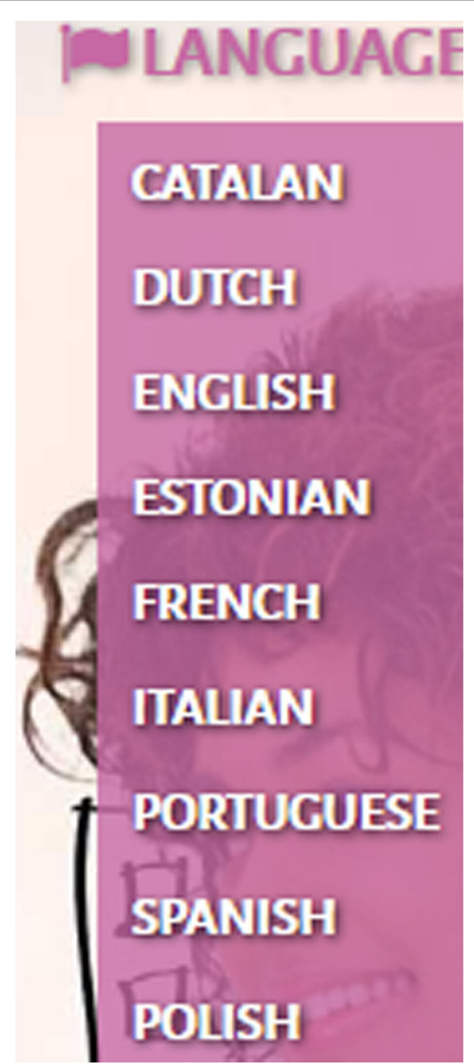

Fig. 12 Drop-down menu for the selection of languages 


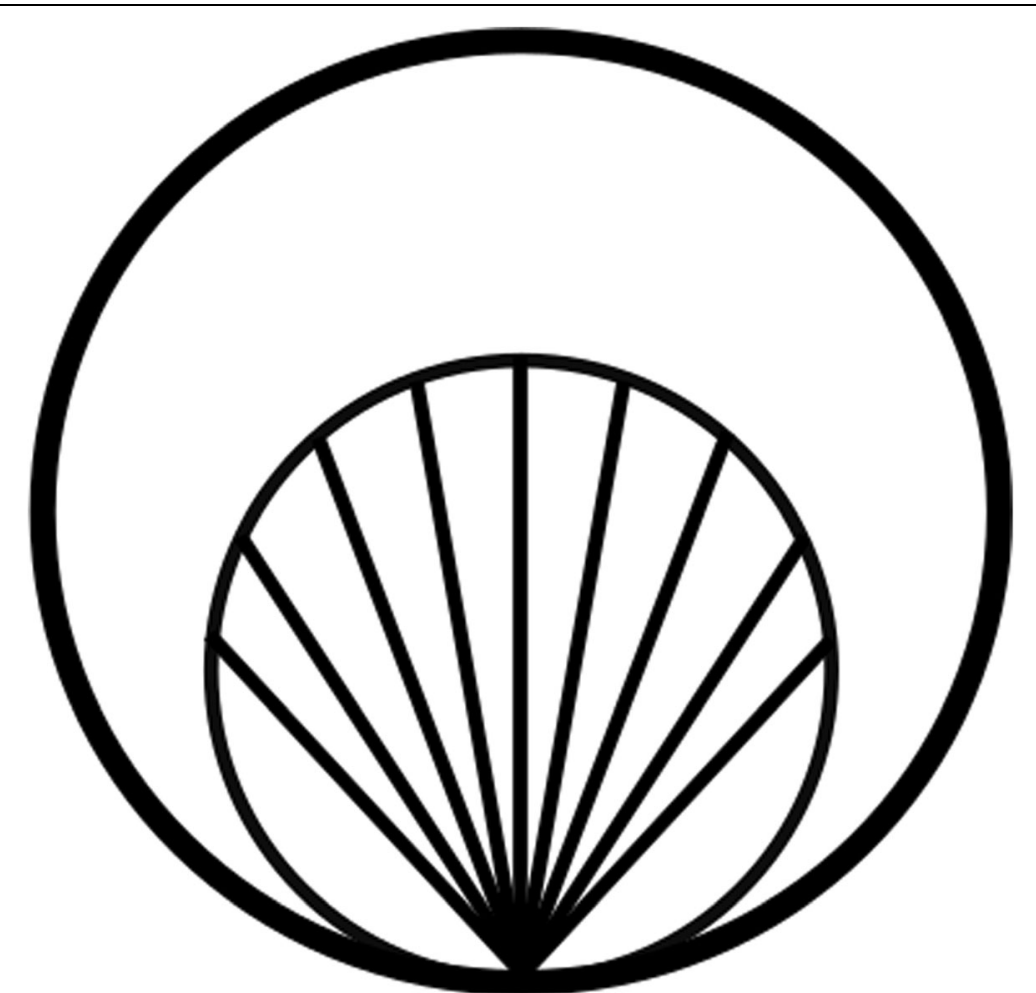

Fig. 13 Legend: The big circle presents the space of the EU, the smaller circle presents regions in EMMA, and lines indicate boundaries between regions in EMMA

Home countries and spaces in between European topologies are further performed on the EMMA interface by specific operations of the integrated translation system. This system operates automatically by using learners' IP-addresses to set the default platform language to the region they are situated in. In addition, it allows learners to select languages manually through the abovementioned drop-down menus and through dropdown menus in each MOOC. However, the system does not translate learners' comments and replies, and merely shows them in the original language they were posted in. As a consequence, interactions between learners mainly occur within a single language, generally their native language. This translation system, thus, seems to favor and aid interactions within linguistic boundaries that, as they closely connect to familiar languages and regional boundaries, enact learners' home country. In contrast to EMMAs objective to traverse European boundaries, the particular workings of the translation system therefore seems to form these boundaries. However, as most MOOCs and some conversations are at least equally available in English, learners can access foreign content or interact through English. Still, since it is impossible to simultaneously see translations to other foreign languages, English does not work as a 'bridge language' that grants access to content in other local languages (Harvey, 2012). Instead, English enacts a boundary as a space in between that connects the linguistically bounded spaces and brings them closer together (Prince, 2015; see Fig. 14).

Slip through Besides the translations, the course materials themselves appear to perform European topologies on the interface. That is, each MOOC articulates the regional origin of the providing university in some specific way, for instance, through 


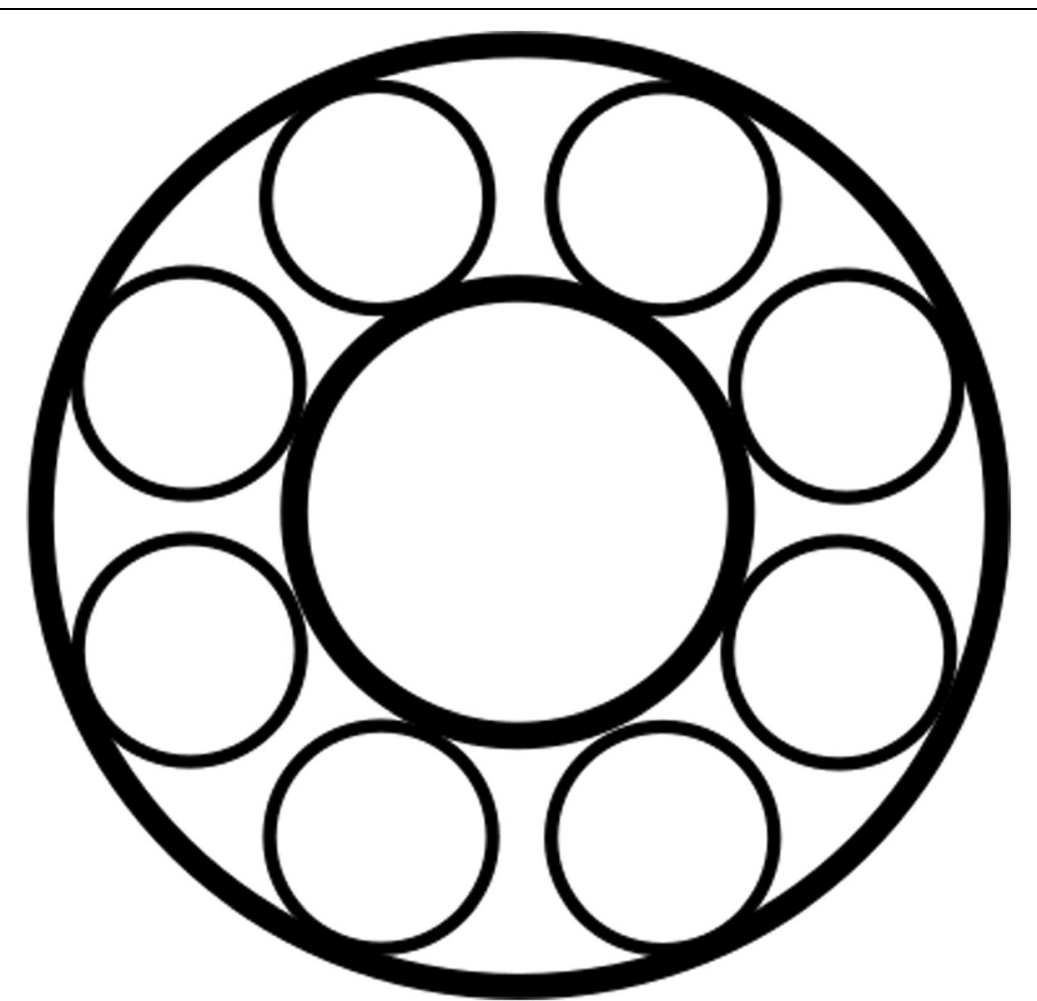

Fig. 14 Legend: Small circles indicate linguistic boundaries, i.e., home countries, which are only connected through the inner circle, which presents English as shared space

cultural examples or non-translated remainders in reading materials. Other examples of boundaries are presented in MOOC assignments where learners were invited to visit a natural setting in a specific region or a website in a regional language. Learners, however, challenged these boundaries as they showed (to be from) places 'outside' the addressed regions in the virtual classroom (see Fig. 15). Thus learners are able to slip through the boundaries and to bring spaces 'inside' EMMA that were initially excluded from the European imaginary (Burridge et al., 2017; Mol \& Law, 1994). The spatiotemporal formation of the course materials is therewith characterized by delimited areas, yet with porous outside borders (Fig. 16).

\section{Beyond-interface: competing, comparing, translating}

Conflated Europeanness European practices beyond the interface emerge out of relations between the EMMA team and its associates. While they largely overlap with network topologies, these topologies are specifically European through new relations. That is, the European nature of these topologies arises out of the relation between the EC, EMMA and other European as well as non-European platforms. The EC formed these relations through practices of competition and comparison specifically because of its ambition to develop platforms that can challenge 'foreign' MOOC providers (European Commission, 2013). Initially, through competition, the EMMA team was urged to focus on a distinct learning audience and to offer something different and 'better' than existing nonEuropean platforms. Yet through comparison, EMMA was evaluated along similar indicators as these other platforms, inviting convergence rather than distinction (e.g., Hartong, 


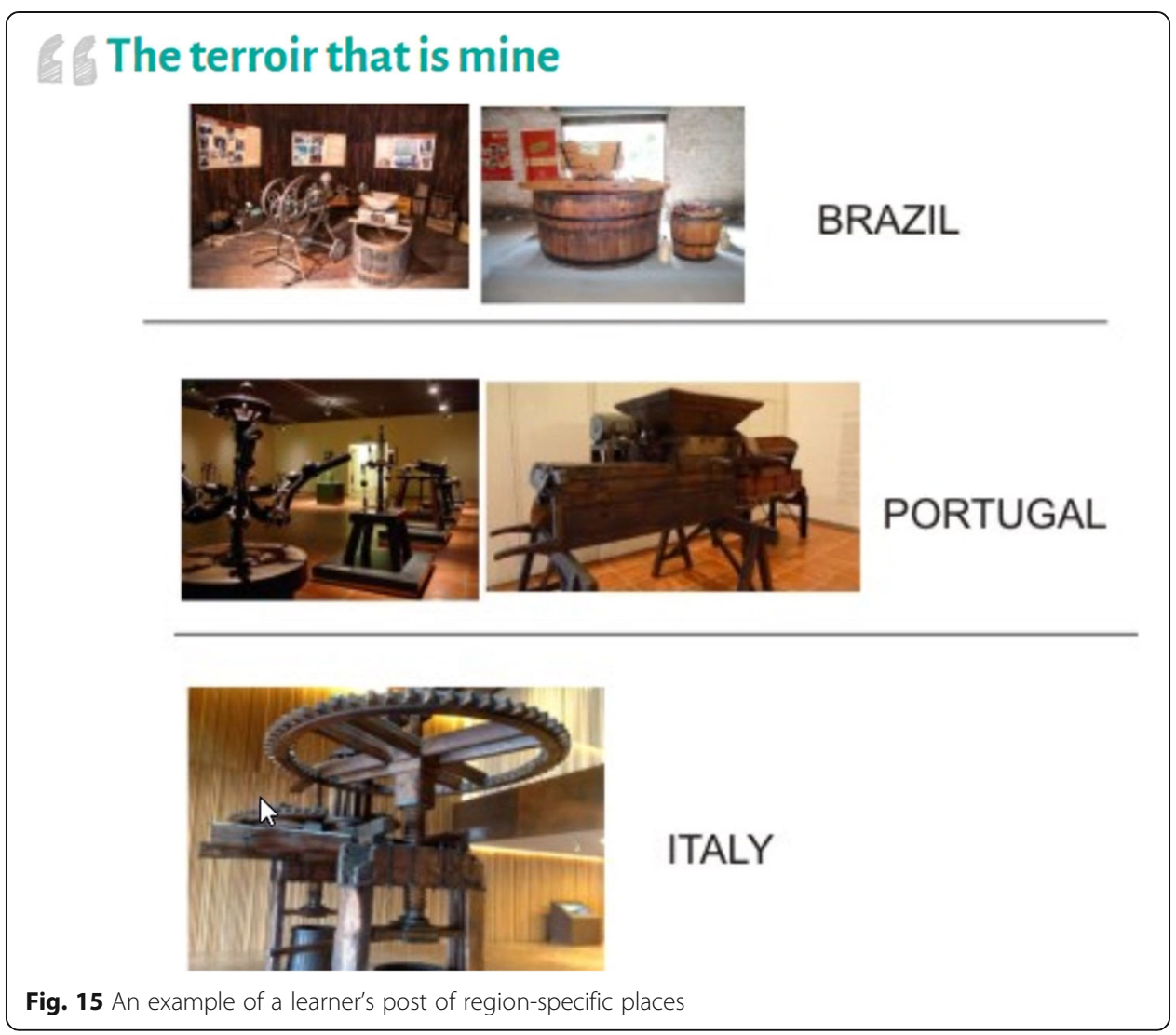

2018). Given these contradictory logics, the EMMA team constantly negotiated between practices that are generally associated with European education and practices of privatization and commodification more specific to North-American MOOC platforms (Van Dijck \& Poell, 2015). For example, they had to balance decisions on the use of multiple European languages or only English text, and publicly funded admittance or efficient business models. Through these comparative and competitive practices, the topology forms a conflated Europeanness that merges values of non-European platforms with a typical European space (Fig. 17).

Linguistic bridges Another European topology comes forward in relations between the EMMA team, the providing universities and partners, thus excluding the EC. This topology operates around a particular structure that connects these associates over their geographical, cultural and linguistic localities and makes them act as a collective (Opitz \& Tellmann, 2015). Central in the practices is the English language which, although appearing on the interface as well, has a specific operation in practices beyond the interface. That is, since the translation system relies on human editing, each provider was urged to translate their learning content particularly in English so that other associates could also develop translations to other languages, i.e., their native language. These English translations therewith enact more than just a space in between, yet rather act as a shared space constructed by and from the different local practices (cf., Prince, 2015; Star, 2010). With respect to the spatiotemporal formation, this means that English enacts a boundary where different localities can meet and interact (Fig. 18). 


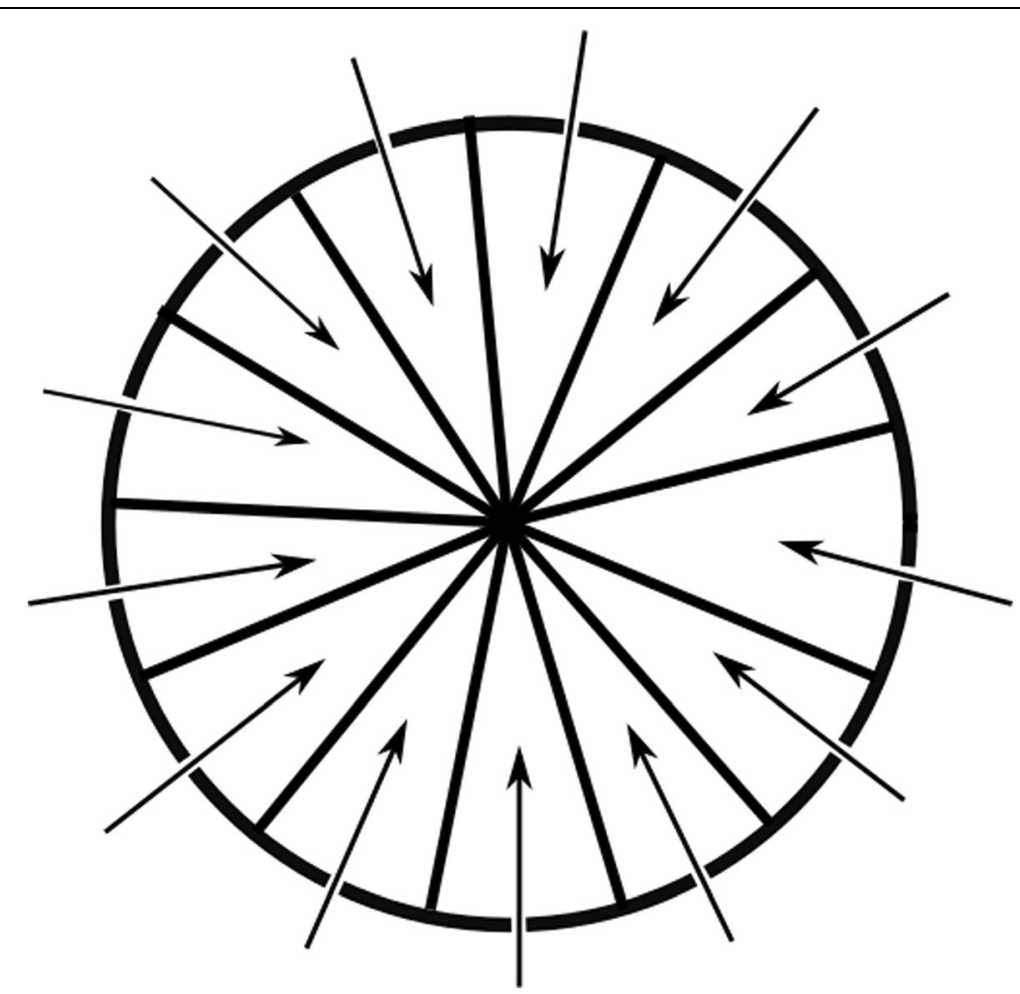

Fig. 16 Legend: Diagonal lines present regions, the small gaps in the outer circle the openings that learners create, the arrows are the way they pass through 'inside'

\section{Data topologies}

Covered by terms as 'research' and 'experimentation', data practices repeatedly surface general debates about openness in education (Weller, 2014). Still, a coherent view on data and how they operate is obscured as they often move through invisible databases and software programs, only to reappear in a visualization (Williamson, 2018). As EMMA initiators equally explicitly refer to data practices (Kerr \& Eradze, 2016), practices within the platform can present how data topologies are configured. As explained here first, oninterface practices produce topologies of self-tracing learners and individualizing spaces and as folds or mirrors of themselves. In a following paragraph, it is described how beyond-interface practices shape traceable learners and produce a mold around these learners. Specific about the data topologies is that they seem to both bound and bind together the different network- access- and European topologies, so that they are realized as complexes of multiple spaces and times.

\section{On-interface: you can see it, because you are in it}

Self-tracing learners On the interface, data topologies especially appear to intersect with other sorts of topologies. One example of such an intersection is visible in the registration procedure which, as previously noted, selects access through EMMA. However, it equally concerns data practices as it generates personal accounts with records of learners' clicks and translates them into personal data visualizations. For example, the PLE shows learners visualizations of vertical timelines with their latest activities, 


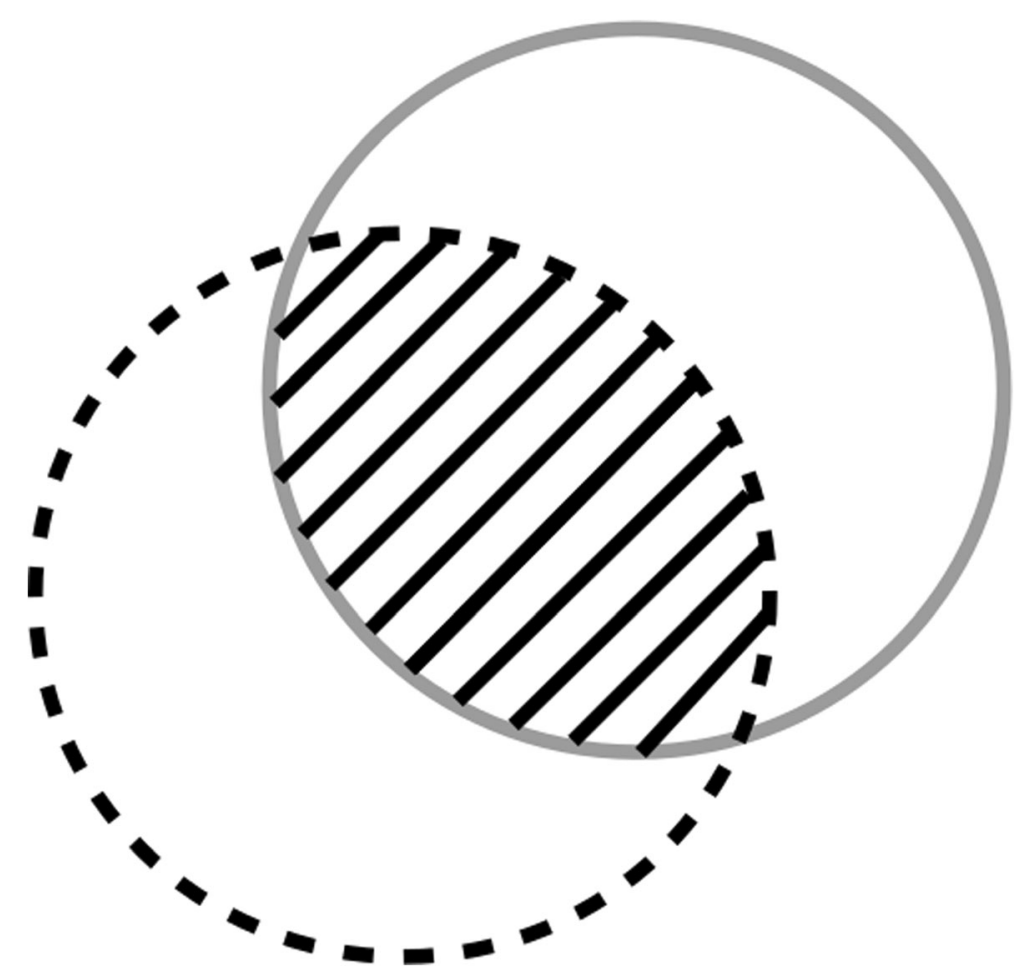

Fig. 17 Legend: The porosity of EMMAs circle indicates her 'openness' to foreign platform models, and the grey circle and the diagonal lines indicate the conflation with non-European platforms

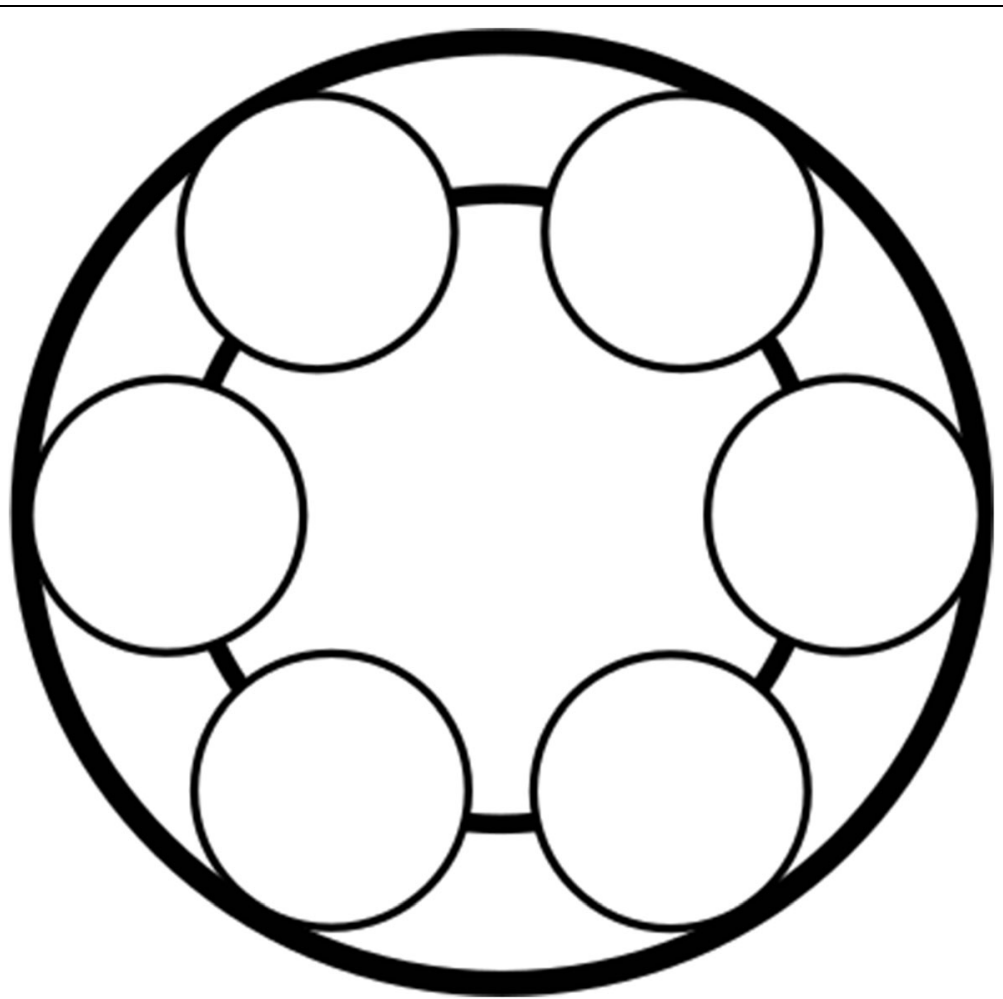

Fig. 18 Legend: The smaller circles indicate associates' languages, the circle they are overlapping is English as the shared space they construct and that connects them 
ring charts on how many units they visited and how many quiz answers were correct and text bars on the amount comments and replies. These visualizations perform an imaginary of self-tracing learners who scrutinize their own actions. Positioning their previous steps like passed achievements on these lines or charts, these visualizations equally present and perform the 'future steps' needed to complete a learning trajectory (Thompson \& Cook, 2017). This is yet another way in which data topologies interweave with access topologies, as these visualizations sketch a map of 'where' and 'when' learners need to go. However, rather than a map that promotes co-presence, this map positions access through EMMA as an individually adjusted path.

Individualizing spaces and folds Besides relating learners to their own actions on EMMA, data practices on the interface equally relate learners to each other in two specific ways. First, the PLE presents aggregate statistics of other learners in the virtual classroom underneath individual statistics, specifically on the same indicators (see Fig. 19). By presenting these statistics together, a topology emerges that brings learners together in a comparative relation. Even without an additional text, this establishes an imaginary of the outcomes that are valued and that one can compete in (Decuypere, 2016). More specifically, it establishes an imaginary of learners who aim to complete more lessons, to get higher quiz grades and post more comments and replies. In a second way, learners are related through data that are integrated into the network visualization presented in the PLE (Fig. 20). This

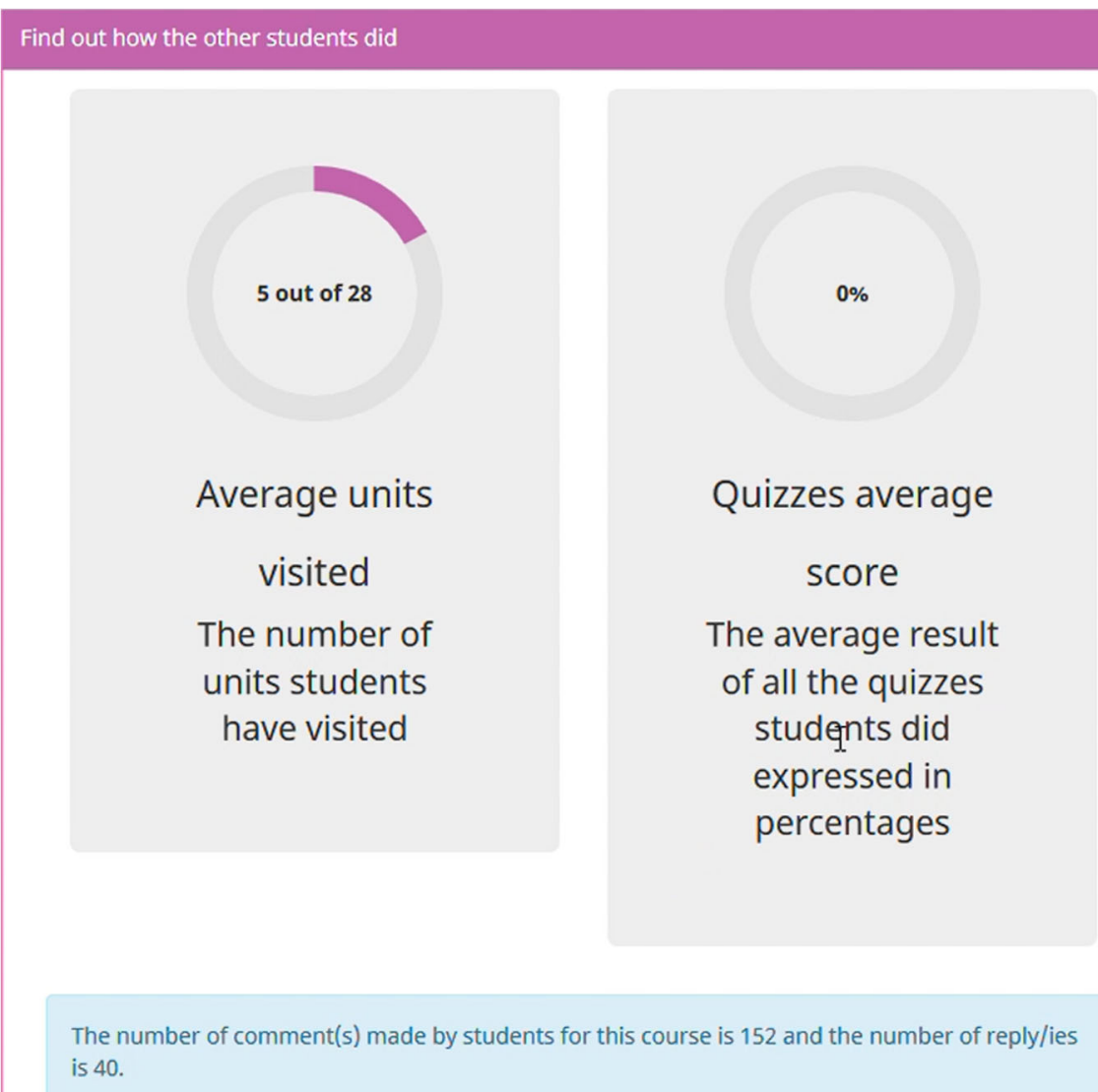

Fig. 19 Ring charts with aggregate statistics and bar on comments and replies 


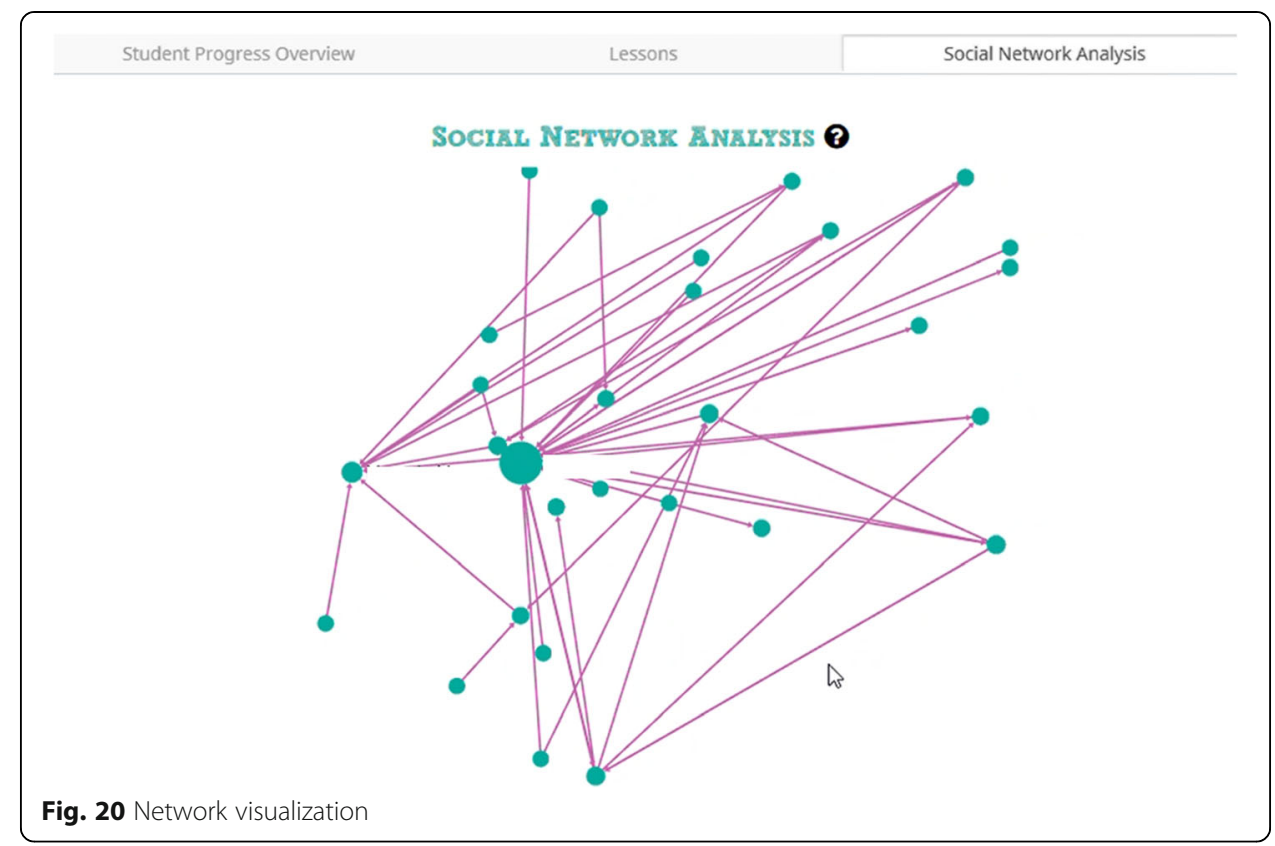

visualization shows learners as nodes in a network and their interactions in the form of line. However, by leaving out other complex details of their learning experience, the data install a particular imaginary on what it means to be in relation to each other (see also Marres, 2012). Here, relations within the EMMA classroom are evaluated by the amount of interactions and the positions of the individual learners in the web of relations rather than what to communicate about and how to maintain relations. By relating learners to each other in this specific way, although counterintuitive, these visualizations establish individualizing spaces (Thompson \& Cook, 2015, 2017; Williamson, 2017). That is, the visualizations make learners individuals as they present their connections with other learners as a way to mirror themselves, rather than to see themselves as integrative part of a learning audience (Fig. 21). Through these visualizations, the data operate as a fold, a material arrangement that produces and reproduces an imaginary of learners and their learning practices by doubling or mirroring them (Deleuze, 1986). This topology does not just operate on the screen of users, but is intricately interwoven with practices beyond the interface.

\section{Beyond-interface: the mold around the fold}

Traceable learners Data practices beyond the interface equally operate by visualizing learners, yet specifically by showing learners and their actions to the EMMA team and her associates while they are on the other side of the interface. That is, the data enact visible indicators for the EMMA team and teachers to interpret learners' behavior on the platform. For example, data show how frequently learners visit particular MOOCs, what kinds of interactions improve their retention or how many minutes they watch a video or read a text before quitting it. This means that data materialize traceable learners whose actions are not only visible to themselves, but also to others (Ruppert, Law, \& Savage, 2013). These traceable learners are composed of data that can be documented through learners' clicks and through survey questions, while it excludes their gazes on the screen or off-line practices like note-taking, scheduling or multi-tasking. 


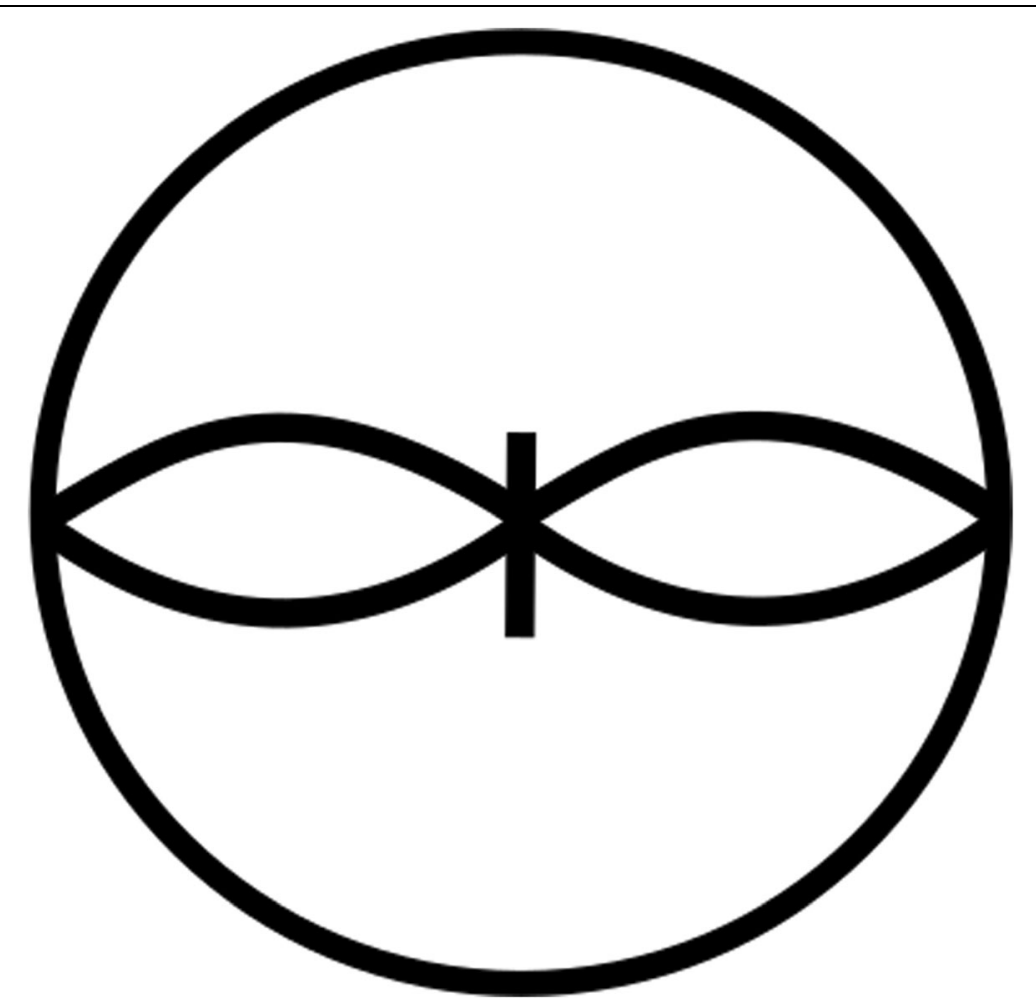

Fig. 21 Legend: In both bows, one (bended) line illustrates data of 'past' achievements while the line back illustrate a path of 'future' steps. One bow presents statistics of personal paths, the other of the aggregate learning audience. The vertical line illustrates how data visualizations work to mirror learners

Thus again, an imaginary of learners is established through a selection of indicators, performing simplified norms regarding what is important for learning on EMMA.

The mold This data topology, the one that makes up an imaginary of learners based on data, is of particular importance for the spatiotemporal configurations of the platform. Namely, it is this imaginary that appears to bind together the different Europeannetwork- and access topologies. This 'binding together' starts with an interaction between data- and European topologies, as data are initially used as a visible passageway to successively inform, transform and again inform the EMMA team and the EC on specific outcomes. They mainly show achievements on the amount of learners on the platform and their retention, aligning with the interest of the EC in expanding and intensifying open and online learning in European countries (European Commission, 2013). Yet data also showed the EMMA team what interaction pages retained the largest learning communities and what hyperlinks eased access through the platform. In turn, this elicited continuous reconfigurations of network and access practices based on what data showed about the best 'fit' between learners' retention and the platform design. This has particular implications for the workings of openness and boundaries in EMMA. First, as the configuration of EMMA focuses on retaining existing learners rather than inviting new learners, boundaries around EMMA do not prioritize to 'open up' to learners who are not on the platform yet. Second, learners are reinforced in their behavior rather than invited to change. For example, they are not impelled to prolong 
their attention span when watching lectures or reflect on the content of their interactions with peers. It implies that data establish a platform that operates as a mold around learners and their behavior. While this mold is enacted through practices beyond the interface, it interacts with the fold topology which forms learners by mirroring them in data visualizations. Together, these interwoven topologies establish a spatiotemporal formation in which a form of the learner is established and stabilized in a '(back)ground' that fits around her or him (Fig. 22). What is specific to data topologies is, then, that they produce the boundaries of different network, access and European topologies by continuously interconnecting (with) them. As we will equally argue in the conclusion, openness can therewith be seen as established in multiplexes of spacestimes, that is, in multiple interconnected forms. This shows how boundaries do not merely imply closures, yet also specific forms of interactions.

\section{Conclusion}

The aim of this study is to examine how an alternative view on openness, one based on the premises of social topology, could provide research and practice relevant starting points to understand and describe openness in MOOCs. Based on these premises, a MOOC platform can be examined as a figure composed of different relations and practices. These configurations of relations and practices establish topologies that, in turn, produce multiple overlapping spaces and times. Furthermore, these topologies operate as imaginaries, which are understood as collective and delimitate norms formed by and forming relations and practices. Boundaries play an important in producing these spaces-times or imaginaries. That is,

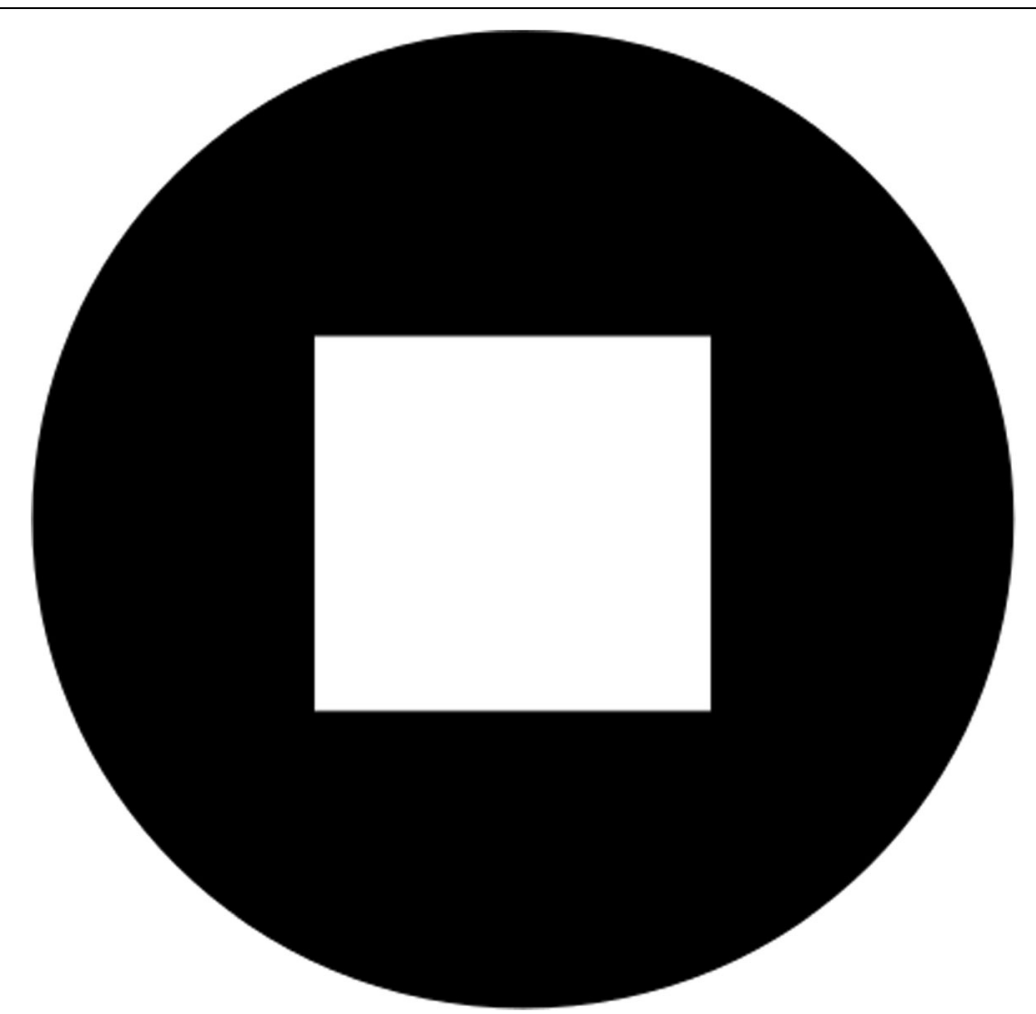

Fig. 22 Legend: The square presents the learner, the black surface presents the platform. Together, they show how the platform forms a mold around the learner and composes a figure, similar to a Gestalt figure/ground 
boundaries make spaces and times by distinguishing what is visible within the particular set of practices, or the topology, and what remains invisible. Yet, these boundaries do not isolate spaces-times, they enable shared spaces between them. Taking together these premises, this means that this view enables to understand openness through the way that boundaries produce collective imaginaries within topologies and how they mediate practices between topologies, rather than through the absence of boundaries.

The premises of this view supported the description of openness in this study in several ways. While this study departed from four classifications of openness, the analysis and its findings particularly present how spaces and times do not operate separately but intersect and interact with each other. That is, within the EMMA platform, practices of network- access- European and data topologies build on each other and are bound together. Second, the findings show that openness is not about an abandonment of boundaries, yet that openness is enacted through particular workings of boundaries. Although boundaries distinguish practices that are visible for learners on the front-end interface or visible on the back-end for those who work on the platform, they also work to mediate between, i.e., to open up, these practices. Moreover, as boundaries determine a visible 'inside' as well as what is 'outside, they produce imaginaries or shared perspectives on where and when a MOOC platform is open and who and what it opens up for. For example, practices in network and access topologies in EMMA produce boundaries that 'open up' multiple ways of moving, both through spaces-times in which teachers and learners come together as in a 'traditional school' (Alhadeff-Jones, 2017; Leaton Gray, 2017) as well as in fragmented and individual frames that are more typical for a digital school (e.g., Hassan, 2017). European practices in EMMA perform boundaries around and between European countries, yet they also create shared spaces that are open to different European regions and bring them closer together (see also Opitz \& Tellmann, 2015). Finally, data practices produce spaces and times that open up as folds that mirror, and molds that shape, learners in the platform. Based on these descriptions, it can be said that openness is mainly enacted by interconnecting multiple forms of spaces and times. This is where the concept 'multiplexes' is introduced.

It is especially this idea of openness as multiplex, that could help as a relevant way of thinking about openness in HE research and practice. First, it serves as a move away from the idea of openness as boundlessness. It shows how HE is not 'opened up' just by introducing new actors and new connections (Ioannou, 2018), yet also foregoes a conclusion that openness is about a disruption of the higher education sector (Knox, 2016). Instead, it describes how boundaries actually can serve specific interconnections that produce a form of openness. Second, rather than fixing a definition or a guideline to improve practice, it inspires to imagine openness in multiple forms. The topologies described in this study therefore merely serve as conceptual tools (Martin \& Secor, 2014), and give researchers and practitioners new reference points for how openness can be conceptualized, operationalized and therewith, scrutinized. This means that HE lecturers and other educational professionals can equally take up the analysis and the findings to consider how they can design their practices in such a way that boundaries produce multiple open learning spaces and times. Therewith, the analysis urges researchers and practitioners to examine what boundaries make visible as well as invisible and thus, what is included and mediated in the constructed imaginaries of openness in MOOCs. 


\section{Endnotes}

${ }^{1}$ Images are retrieved from the EMMA platform: https://platform.europeanmoocs. eu/, https://platform.europeanmoocs.eu/MOOCs, and pages related to a personal account. Names are anonymized by covering them with white or black marker and personal photos are covered with the default 'anonymous' picture on EMMA.

${ }^{2}$ The original term used by interviewees to refer to the partners, providers and the EC, was 'stakeholders'. This is translated into a term that suits the conceptual repertoire of social topology.

\section{Abbreviations}

EC: European Commission; EMMA: European Multiple MOOC Aggregator; HE: Higher education; MOOC: Massive Open Online Course; PLE: Personal learning environment

\section{Acknowledgements}

Special thanks to the interviewees for the warm welcomes in their offices or over distance communication and for their input on the 'beyond interface' practices as described in this report.

\section{Authors' contributions}

The first author is responsible for the literature review, data collection and analysis. Both authors closely collaborated in the development of the conceptual, theoretical and methodological framework as well as in the preparation of the manuscript and hence, both have agreed on the final conclusions.

\section{Funding}

This research did not receive a specific grant from any funding agency in the public, commercial or non-profit sectors.

\section{Availability of data and materials}

The datasets generated and analyzed during the current study are not publicly available, considering the confidential nature of the data, but they are available from the corresponding author on reasonable request.

\section{Competing interests}

The authors declare that they have no competing interests.

Received: 20 February 2019 Accepted: 28 May 2019

Published online: 16 July 2019

\section{References}

Alhadeff-Jones, M. (2017). Time and the rhythms of emancipatory education. Abingdon: Routledge.

Baker, F. W. (2017). An alternative approach: Openness in education over the last 100 years. TechTrends, 61, 130-140. https:// doi.org/10.1007/s11528-016-0095-7.

Bayne, S., Gallagher, M. S., \& Lamb, J. (2014). Being "at" university: The social topologies of distance students. Higher Education 67(5), 569-583. https://doi.org/10.1007/s10734-013-9662-4.

Bayne, S., Knox, J., \& Ross, J. (2015). Open education: The need for a critical approach. Learning, Media and Technology, 40(3), 247-250. https://doi.org/10.1080/17439884.2015.1065272.

Bødker, H., \& Brügger, N. (2018). The shifting temporalities of online news: The Guardian's website from 1996 to 2015. Journalism, 19(1), 56-74. https://doi.org/10.1177/1464884916689153.

Burridge, A., Gill, N., Kocher, A., \& Martin, L. (2017). Polymorphic borders. Territory, Politics, Governance, 5(3), 239-251. https:// doi.org/10.1080/21622671.2017.1297253.

Büttner, S. M., Leopold, L., Mau, S., \& Posvic, M. (2015). Professionalization in EU policy-making? The topology of the transnational field of EU affairs. European Societies, 17(4), 569-592. https://doi.org/10.1080/14616696.2015.1072229.

Callon, M., Lascoumes, P., \& Barthe, Y. (2009). Acting in an uncertain world. An essay on technical democracy. Cambridge: The MIT Press.

Cormier, D. (2008). Rhizomatic education: Community as curriculum. Innovate: Journal of Online Education, 4(5) Retrieved from https://nsuworks.nova.edu/innovate/vol4/iss5/2/. Accessed 10 Oct 2018

Crea, T. M., \& Sparnon, N. (2017). Democratizing education at the margins: Faculty and practitioner perspectives on delivering online tertiary education for refugees. International Journal of Educational Technology in Higher Education, 14(43), 1-19. https://doi.org/10.1186/s41239-017-0081-y.

Dahlberg, G. M., \& Bagga-Gupta, S. (2017). Understanding glocal learning spaces. An empirical study of languaging and transmigrant positions in the virtual classroom. Learning, Media and Technology, 39(4), 468-487. https://doi.org/10.1080/ 17439884.2014.931868

Decuypere, M. (2016). Diagrams of Europeanization: European education governance in the digital age. Journal of Education Policy, 31(6), 851-872. https://doi.org/10.1080/02680939.2016.1212099.

Decuypere, M., \& Simons, M. (2016). Relational thinking in education: topology, sociomaterial studies, and figures. Pedagogy Culture and Society, 24(3), 371-386. https://doi.org/10.1080/14681366.2016.1166150.

Decuypere, M. (2018). Open Education platforms: Theoretical ideas, digital operations and the figure of the open learner. European Educational Research Journal. Advance online publication. https://doi.org/10.1177/1474904118814141.

Deleuze, G. (1986). Foucault, (7th ed., ). Minneapolis: University of Minnesota Press.

Downes, S. (2007). Models for sustainable open educational resources. Interdisciplinary Journal of Knowledge and Learning Objects, 3, 29-44. Retrieved from https://www.learntechlib.org/p/44796/. 
Edwards, R. (2015). Knowledge infrastructures and the inscrutability of openness in education. Learning, Media and Technology, 40(3), 251-264. https://doi.org/10.1080/17439884.2015.1006131.

European Commission (2013). Opening up education: Innovative teaching and learning for all through new technologies and open educational resources. Brussels: Retrieved from http://eur-lex.europa.eu/legal-content/EN/TXT/PDF/?uri=CELEX: 52013DC0654\&from=EN. Accessed 22 Jan 2018

Farrow, R. (2017). Open education and critical pedagogy. Learning, Media and Technology, 42(2), 130-146. https://doi.org/10. 1080/17439884.2016.1113991.

Funes, M., \& Mackness, J. (2018). When inclusion excludes: A counter narrative of open online education. Learning, Media and Technology, 34(2), 119-138. https://doi.org/10.1080/17439884.2018.1444638.

Hartong, S. (2018). Towards a topological re-assemblage of education policy? Observing the implementation of performance data infrastructures and 'centers of calculation' in Germany. Globalisation, Societies and Education, 16(1), 134-150. https:// doi.org/10.1080/14767724.2017.1390665.

Harvey, D. (1989). The condition of postmodernity: An enquiry into the origins of cultural change. Oxford: Blackwell Publishers.

Harvey, P. (2012). The topological quality of infrastructural relation: An ethnographic approach. Theory, Culture \& Society, 29(5), 76-92. https://doi.org/10.1177/0263276412448827.

Hassan, R. (2017). The worldly space: The digital university in network time. British Journal of Sociology of Education, 38(1), $72-82$. https://doi.org/10.1080/01425692.2016.1234364.

Ioannou, O. (2018). Opening up design studio education using blended and networked formats. International Journal of Educational Technology in Higher Education, 15(47), 1-16. https://doi.org/10.1186/s41239-018-0129-7.

Jasanoff, S. (2015). Future imperfect: Science, technology, and the imaginations of modernity. In S. Jasanoff, \& K. Sang-Hyun (Eds.), Dreamscapes of modernity. Sociotechnical imaginaries and the fabrication of power, (pp. 1-47). Chicago: The University of Chicaco Press.

Kerr, R., \& Eradze, M. (2016). MOOCs: A multi-faceted phenomenon. Italian Journal of Educational Technology, 24(2), 126-129. https://doi.org/10.17471/2499-4324/897.

Kerr, R., \& Merciai, I. (2015). EMMA: Towards multicultural learning. The EuroCALL Review, 23(2), 53-58. https://doi.org/10.4995/ eurocall.2016.5696.

Kitchin, R., \& Dodge, M. (2011). Code / space: Software and everyday life. Cambridge: MIT Press.

Knox, J. (2013). Five critiques of the open educational resources movement. Teaching in Higher Education, 18(8), 821-832. https://doi.org/10.1080/13562517.2013.774354.

Knox, J. (2016). Posthumanism and the MOOC: Opening the subject of digital education. Studies in Philosophy and Education, 35(3), 305-320. https://doi.org/10.1007/s11217-016-9516-5.

Lash, S. (2012). Deforming the figure: Topology and the social imaginary. Theory, Culture \& Society, 29(5), 261-287. https://doi. org/10.1177/0263276412448829.

Latour, B. (1986). Visualization and cognition: Thinking with eyes and hands. Knowledge and Society: Studies in the Sociology of Culture Past and Present, 6, 1-40.

Leaton Gray, S. (2017). The social construction of time in contemporary education: Implications for technology, equality and Bernstein's 'conditions for democracy'. British Journal of Sociology of Education, 38(1), 60-71. https://doi.org/10.1080/ 01425692.2016 .1234366

Marres, N. (2012). On some uses and abuses of topology in the social analysis of technology (or the problem with smart meters). Theory, Culture \& Society, 29(5), 288-310. https://doi.org/10.1177/0263276412454460.

Martin, A.D. \& Kamberelis, G., G. (2013). Mapping not tracing: Qualitative educational research with political teeth. International Journal of Qualitative Studies in Education, 26(6), 668-679. https://doi.org/10.1080/09518398.2013. 788756.

Martin, L., \& Secor, A. J. (2014). Towards a post-mathematical topology. Progress in Human Geography, 38(3), 420-438. https:// doi.org/10.1177/0309132513508209.

Mezzadra, S., \& Neilson, B. (2012). Between inclusion and exclusion: On the topology of global space and borders. Theory, Culture \& Society, 29(5), 58-75. https://doi.org/10.1177/0263276412443569.

Mol, A., \& Law, J. (1994). Regions, networks and fluids: Anaemia and social topology. Social Studies of Science, 24, 641-671. https://doi.org/10.1177/030631279402400402.

November, V., Camacho-Hübner, E., \& Latour, B. (2010). Entering a risky territory: Space in the age of digital navigation. Environment and Planning D: Society and Space, 28(4), 581-599. https://doi.org/10.1068/d10409.

Oliver, M. (2015). From openness to permeability: Reframing open education in terms of positive liberty in the enactment of academic practices. Learning, Media and Technology, 40(3), 365-384. https://doi.org/10.1080/17439884.2015.1029940.

Opitz, S., \& Tellmann, U. (2015). Europe as infrastructure: Networking the operative community. South Atlantic Quarterly, 114(1), 171-190. https://doi.org/10.1215/00382876-2831356.

Parisi, L. (2012). Digital design and topological control. Theory, Culture \& Society, 29(5), 165-192. https://doi.org/10.1177/ 0263276412443568

Peters, M. A. (2014). Openness and the intellectual commons. Open Review of Educational Research, 1(1), 1-7. https://doi.org/ 10.1080/23265507.2014.984975.

Prince, R. (2015). The spaces in between: Mobile policy and the topographies and topologies of the technocracy. Environment and Planning D: Society and Space, 34(3), 420-437. https://doi.org/10.1177/0263775815618401.

Ross, J., \& Sheail, P. (2017). The 'campus imaginary': Online students' experience of the masters dissertation at a distance. Teaching in Higher Education, 22(7), 839-854. https://doi.org/10.1080/13562517.2017.1319809.

Ruppert, E. (2012). The governmental topologies of database devices. Theory, Culture \& Society, 29(5), 116-136. https://doi.org/ $10.1177 / 0263276412439428$.

Ruppert, E., Law, J., \& Savage, M. (2013). Reassembling social science methods: The challenge of digital devices. Theory, Culture \& Society, 30(4), 22-46. https://doi.org/10.1177/0263276413484941.

Sha, X. W. (2012). Topology and morphogenesis. Theory, Culture \& Society, 29(4/5), 220-246. https://doi.org/10.1177/0263276412443570

Sheail, P. (2018). The digital university and the shifting time-space of the campus. Learning, Media and Technology, 43(1), 56-69. https://doi.org/10.1080/17439884.2017.1387139.

Sørensen, E. (2009). The materiality of learning. New York: Cambridge University Press. 
Srinivasan, P. (2017). What we see is what we choose: Seers and seekers with diversity. Pedagogy, Culture and Society, 25(2), 293-307. https://doi.org/10.1080/14681366.2016.1255243.

Star, S. L. (1999). The ethnography of infrastructure. American Behavioral Scientist, 43(4), 377-391. https://doi.org/10.1177/ 00027649921955326.

Star, S. L. (2010). This is not a boundary object: Reflections on the origin of a concept. Science Technology and Human Values, 35(5), 601-617. https://doi.org/10.1177/0162243910377624.

Star, S. L., \& Griesemer, J. R. (1989). Institutional ecology, "translations" and boundary objects: Amateurs and professionals in Berkeley's Museum of Vertebrate Zoology, 1907-39. Social Studies of Science, 19, 387-420. https://doi.org/10.1177/030631289019003001.

Star, S. L., \& Ruhleder, K. (1996). Steps toward design an ecology and access of infrastructure. Information Systems Research, 7(1), 111-134. https://doi.org/10.1287/isre.7.1.111

Stracke, C. M. (2018). How can Open Education improve learning quality and achieve impact for learners, organizations and in society? In T. Amiel (Ed.), Utopias and Dystopias in education. Sao Paulo: UNICAMP.

Thompson, G., \& Cook, I. (2015). Becoming-topologies of education: Deformations, networks and the database effect. Discourse: Studies in the Cultural Politics of Education, 36(5), 732-748. https://doi.org/10.1080/01596306.2014.890411.

Thompson, G., \& Cook, I. (2017). The logic of data-sense: Thinking through learning personalisation. Discourse: Studies in the Cultural Politics of Education, 38(5), 740-754. https://doi.org/10.1080/01596306.2016.1148833.

Tucker, I., \& Goodings, L. (2014). Mediation and digital intensities: Topology, psychology and social media. Social Science Information, 53(3), 277-292. https://doi.org/10.1177/0539018414525693.

Van Dijck, J., \& Poell, T. (2015). Higher education in a networked world: European responses to U.S. MOOCs. International Journal of Communication, 9, 2674-2692. Retrieved from https://ssrn.com/abstract=2645629

Weller, M. (2014). The Battle for open: How openness won and why it doesn't feel like victory. London: Ubiquity Press.

Weller, M., Jordan, K., Devries, I., \& Rolfe, V. (2018). Mapping the open education landscape: Citation network analysis of historical open and distance education research. Open Praxis, 10(2), 109-126. https://doi.org/10.5944/openpraxis.10.2.822.

Williamson, B. (2017). Decoding ClassDojo: Psycho-policy, social-emotional learning and persuasive educational technologies. Learning, Media and Technology, 42(4), 440-453. https://doi.org/10.1080/17439884.2017.1278020.

Williamson, B. (2018). The hidden architecture of higher education: Building a big data infrastructure for the 'smarter university'. International Journal of Educational Technology in Higher Education, 15(1), 1-26. https://doi.org/10.1186/s41239018-0094-1.

\section{Publisher's Note}

Springer Nature remains neutral with regard to jurisdictional claims in published maps and institutional affiliations.

\section{Submit your manuscript to a SpringerOpen ${ }^{\circ}$ journal and benefit from:}

- Convenient online submission

- Rigorous peer review

- Open access: articles freely available online

- High visibility within the field

- Retaining the copyright to your article

Submit your next manuscript at $\boldsymbol{\nabla}$ springeropen.com 